\title{
INTEGRATION IN A CONVEX LINEAR TOPOLOGICAL SPACE*
}

\author{
BY \\ R. S. PHILLIPS
}

This paper is concerned with a theory of integration for functions with values in a convex linear topological space. We consider an integral which is essentially an extension to this general space of the integral studied by Garrett Birkhoff [1] in a Banach space. By imposing different convex neighborhood topologies on a Banach space, we obtain as instances of our integral those defined by Birkhoff [1], Dunford [2], Gelfand [3], and Pettis [4].

Let $f(s)$ be a function on an abstract set $S$ to the real numbers, and let $\alpha(\sigma)$ be a nonnegative measure function on an additive family of subsets $\delta$ of $S$. A necessary and sufficient condition for the Lebesgue integral to exist is that for each $e>0$ there exist a partition $\Delta_{e}$ of $S$ into a denumerable set of sets $\left(\sigma_{i}\right)$ such that for any two orderings of these sets, $\left(\sigma_{i}{ }^{1}\right)$ and $\left(\sigma_{i}{ }^{2}\right)$,

$$
\left|\lim \sup _{n} \sum^{n} \alpha\left(\sigma_{i}{ }^{1}\right) \cdot \sup _{s_{i} \varepsilon \sigma_{i}}{ }^{1} f\left(s_{i}\right)-\lim _{n} \inf \sum^{n} \alpha\left(\sigma_{i}{ }^{2}\right) \cdot \inf _{s_{i} \varepsilon \sigma_{i}}{ }^{2} f\left(s_{i}\right)\right|<e .
$$

We have introduced upper and lower limits in order to stress the fact that unconditional convergence is an unnecessary concept in the definition of the Lebesgue integral. In fact by avoiding unconditional convergence we have been able to extend this integral to a linear convex topological space. This further permits a simplification in the concepts involved.

In the first section we consider a few relations between linear continuous operations and convex neighborhoods which have been found useful in the study of the integral. In $\$ 2$ the integral is defined and some essential properties of unconditional summability are established. This is followed in $\S 3$ by a discussion of the basic properties of the integral. The integral of an integrable function $x$ is an absolutely continuous and completely additive set function depending linearly on $x$. It is found in $\$ 4$ that the integrability conditions can be relaxed when the space satisfies a certain type of completeness condition. A demonstration that the linear continuous transform of an integrable function is itself integrable occupies $\$ 5$. Section 6 treats convergent sequences of integrable functions, while $\$ 7$ deals with relations to other integrals. A theorem on differentiation is proved in $\$ 8$. In $\$ 9$ an application is made of this theory to an existence theorem in differential equations. Finally in $\$ 10$

\footnotetext{
* Presented to the Society, April 15, 1939; received by the editors June 19, 1939.
} 
some examples are given to demonstrate properties of the integral. Example 10.2 shows that the Birkhoff and Pettis integrals are different. As a consequence our development leads to the definition of a class of integrals for the theory of integration on a Banach space.

1. On convex linear topological spaces. We will restrict ourselves in this paper to the type of convex linear topological space $X$ defined by Kolmogoroff $[5$, p. 29] to satisfy the following axioms:

1. $X$ is a linear space relative to real numbers $[6$, p. 26].

2. There exists an operation of closure, $\bar{B}$, for any subset $B$ of $X$ with the following properties:

2a. If $B$ consists of a single element of $X$, then $\bar{B}=B$.

2b. $\bar{B}=\bar{B}$.

2c. $\overline{B+C}=\bar{B}+\bar{C}$ ( + stands for set addition).

3. Addition of elements and multiplication by numbers are continuous.

4. There exists an equivalent neighborhood system of convex* neighborhoods $U$. Kolmogoroff has shown that Axioms 1, 2, 3 are sufficient to make $X$ a regular Hausdorff space with a uniform topology. $\dagger$ We will therefore use only neighborhoods of the null vector.

In a convex space pseudo-norms have been defined by von Neumann [7, pp. 18-19] as follows: Let $\|x\|_{U}^{+}$equal the greatest lower bound of $a>0$ such that $x \varepsilon a U$, and let $\|x\|_{U}=\max \left[\|x\|_{U}^{+},\|-x\|_{U}^{+}\right]$. He has established the following properties by arguments that are applicable to our space:

$$
\|x+y\|_{U} \leqq\|x\|_{U}+\|y\|_{U}, \quad\|a x\|_{U}=|a| \cdot\|x\|_{U}, \quad\|\theta\|_{U}=0,
$$

$\|x\|_{U}$ is a continuous function of $x$, and the sets $\|x\|_{U}<\delta, U \varepsilon V, \delta>0$ form a complete system of neighborhoods for $\theta$. The norm has the additional property: $\|x\|=0$ if and only if $x=\theta$. This is not a property of the pseudo-norm. In the remainder of this paper we will use the system of neighborhoods defined by the pseudo-norms and designate by $U$ the set of all $x$ such that $\|x\|_{U}<1$. Hence $U_{1} \supset U_{2}$ is equivalent to the statement that $\|x\|_{U_{2}}<1$ implies $\|x\|_{U_{1}}<1$. Since the pseudo-norm is homogeneous and continuous, this is in turn equivalent to $\|x\|_{U_{1}} \leqq\|x\|_{U_{2}}$.

Let us now set up a one-to-one correspondence between the set of all linear continuous operations $\ddagger$ on $X$ and an abstract class $T$ of the same cardinal power; say, $\bar{x}_{t}$ corresponds to $t \varepsilon T$. We proceed to prove that the neighborhoods of $X$ can be defined by linear continuous operations on $X$.

* A set $B$ is convex if, whenever $x, y \in B, a, b \geqq 0$, and $a+b=1$, then $a x+b y \varepsilon B$.

$\dagger X$ is said to have a uniform topology if, when $\mathcal{V}(x)$ and $\mathcal{V}(\theta)$ are the neighborhood systems for $x$ and $\theta$ respectively, $x+\mathcal{U}(\theta)$ is equivalent to $\mathcal{U}(x)$.

$\ddagger$ The term "operation" is used for a numerical function. 
THEOREM 1.1. For each $U \varepsilon \cup$ there exists a subset $T_{U}$ of $T$ such that $\left[x|x \varepsilon X,| \bar{x}_{t}(x) \mid<1\right.$ for every $\left.t \varepsilon T_{U}\right] \equiv\left[x \mid x \varepsilon X,\|x\|_{U}<1\right]$. $^{*}$

Wehausen [8, p. 162, Theorem 8] has demonstrated that given any $x_{0} \varepsilon X$ and $U \varepsilon V$, there exists a linear continuous operation $\bar{x}_{0}$ on $X$ with the property that $\left|\bar{x}_{0}(x)\right| \leqq\|x\|_{U}$ and $\bar{x}_{0}\left(x_{0}\right)=\left\|x_{0}\right\|_{U}$. To each $x \varepsilon X$ corresponds in this fashion $\bar{x}_{t_{x}}$. To the set of $x \varepsilon X$ for which $\|x\|_{U}=1$ will then correspond a subset $T_{U} \equiv\left[t_{x}\right]$ of $T$. For $t \varepsilon T_{U},\left|\bar{x}_{t}(x)\right| \leqq\|x\|_{U}$, and hence $\left[x|x \varepsilon X,| \bar{x}_{t}(x) \mid<1\right.$ for every $\left.t \varepsilon T_{U}\right] \supset\left[x \mid x \varepsilon X,\|x\|_{U}<1\right]$. Further if $\left|\bar{x}_{t}(x)\right|<1$ for every $t \varepsilon T_{U}$, then either $\|x\|_{U}=0$, or $\|x\|_{U} \neq 0$, in which case there exist $t_{a x} \varepsilon T_{U}$ and $a>1$ such that $\left|\bar{x}_{t_{a x}}(a x)\right|=1=\|a x\|_{U}$ and $\|x\|_{U}=1 /|a|<1$. The inclusion therefore holds both ways.

We will use interchangeably the three symbols $U,\left[x \mid x \varepsilon X,\|x\|_{U}<1\right]$, and $\left[x|x \varepsilon X,| \bar{x}_{t}(x) \mid<1\right.$ for every $\left.t \varepsilon T_{U}\right]$ since they represent the same subset of $X$. The last expression can be considered as an analysis of the neighborhood $U$ just as the neighborhoods $U$ furnish a decomposition of the neighborhood system $\mho$.

It is instructive to investigate the relation between the set of all linear continuous operations on $X$ and that subset used to define the neighborhood system. This can be conveniently described by means of the algebraic notion of a factor group. Essentially the factor group permits us to study a linear convex topological space by means of a set of linear normed vector spaces.

If $\Theta$ is a closed linear manifold in $X$, then, being a subgroup of $X$, $\Theta$ defines a factor group $Y$ whose elements $y$ correspond biuniquely to the cosets. $Y$ is again a linear space. Now for a given $U \varepsilon v$ we will define $\Theta_{U} \equiv\left[x \mid x \varepsilon X,\|x\|_{U}=0\right]$ or what is equivalent $\Theta_{U} \equiv\left[x \mid x \varepsilon X, \bar{x}_{t}(x)=0\right.$ for every $\left.t \varepsilon T_{U}\right]$. Then as $\Theta_{U}$ is linear and closed, it defines the factor group $Y_{U}$. We define a norm on $Y_{U}$ to be $\|y\|=\|x\|_{U}$ where $x$ is any element of the coset $y$. This is clearly independent of the particular $x \varepsilon \operatorname{coset} y$ used and satisfies all of the properties of a norm. The transformation $y=T(x)$, where $x \varepsilon \operatorname{coset} y$, is a linear continuous transformation on $X$ to $Y_{U}$ with its norm topology. A linear continuous operation $\bar{y} \varepsilon \bar{Y}_{U}$, the space adjoint to $Y_{U}$, defines a linear continuous operation $\bar{x}=\bar{T}(\bar{y})$, namely $\bar{x}(x)=\bar{y}(y)$ where $x \varepsilon \operatorname{coset} y$. We will use this correspondence in the statement of the following theorem:

THEOREM 1.2. The set of all linear continuous operations on $X$ is the linear extension of the set of operations which correspond to the adjoint spaces $\bar{Y}_{U}$ for all $U \varepsilon v$.

If $\bar{y} \varepsilon \bar{Y}_{U}$, then $|\bar{x}(x)|=|\bar{y}(y)| \leqq\|\bar{y}\| \cdot\|y\|=\|\bar{y}\| \cdot\|x\|_{U}$. In other words

* We will designate by $[y \mid P]$ the class of elements $y$ with the properties $P$. 
$|\bar{x}(x)|<e$ if $x \varepsilon e U /\|\bar{y}\|$. On the other hand if $\bar{x}$ is continuous on $X$, then by a theorem due to Wehausen [8, pp. 162-163, Theorem 9], there exists a $U$ \& $V$ and a positive constant $M$ for which $|\bar{x}(x)| \leqq M \cdot\|x\|_{U}$. The correspondence $\bar{y}(y)=\bar{x}(x)$ where $x \varepsilon$ coset $y$ makes $\bar{x}$ correspond to an element of $\bar{Y}_{U}$.

THEOREM 1.3. If the set $\left[\bar{x}_{t} \mid t \varepsilon T_{U}\right]$ has $n$ and only $n$ linearly independent elements, then $Y_{U}$ has $n$ and only $n$ linearly independent elements.

Suppose first that there exist $n+1$ linearly independent vectors $y_{1}, \cdots, y_{n+1}$ in $Y_{U}$, and that $\bar{x}_{1}, \cdots, \bar{x}_{n} \varepsilon\left[\bar{x}_{t} \mid t \varepsilon T_{U}\right]$ are linearly independent. If $\bar{y}_{t}$ corresponds to $\bar{x}_{t}$, then the system of $n$ homogeneous linear equations $\sum_{i=1}^{n+1} a_{i} \bar{y}_{t}\left(y_{i}\right)=0(t=1, \cdots, n)$ has a non-trivial solution $\left\{a_{i}\right\}$. Let $x_{i}$ be an element of coset $y_{i}$. Since $\sum_{i=1}^{n+1} a_{i} \bar{x}_{t}\left(x_{i}\right)=\sum_{i=1}^{n+1} a_{i} \bar{y}_{t}\left(y_{i}\right)=0(t=1, \cdots, n)$ and since any other $\bar{x}_{t}$ for $t \varepsilon T_{U}$ is a linear combination of $\bar{x}_{1}, \cdots, \bar{x}_{n}$, it follows that $\bar{x}_{t}\left(\sum_{i=1}^{n+1} a_{i} x_{i}\right)=0$ for all $t \varepsilon T_{U}$. Hence $\sum_{i=1}^{n+1} a_{i} x_{i} \varepsilon \Theta_{U}$ which is contrary to our supposition. On the other hand, if there do not exist $n$ linearly independent elements in $Y$, then some linear combination of every set of $n$ elements of $X$ belongs to $\dot{\Theta}_{U}$. Hence for every set $x_{1}, \cdots, x_{n}$, the $n$ rowed determinant $\left|\bar{x}_{t}\left(x_{j}\right)\right|=0(t, j=1, \cdots, n)$. Let $r$ be the largest rank attained by $\left|\bar{x}_{t}\left(x_{j}\right)\right|$ for any choice of $\left\{x_{j}\right\}$-say $\left|\bar{x}_{t}\left(x_{j}\right)\right| \neq 0(t, j=1, \cdots, r)$. Then $\left|\bar{x}_{t}\left(x_{j}\right)\right|=0$ $(t, j=1, \cdots, r+1)$ for any $x_{r+1} \varepsilon X$ and hence there exist $\left\{c_{t}\right\}$ such that $\sum_{t=1}^{r+1} c_{t} \bar{x}_{t}(x)=0$ for all $x \varepsilon X$. But this means that $\bar{x}_{1}, \cdots, \bar{x}_{r+1}$ are linearly dependent.

COROLLARY 1.4. If each of the sets $\left[\bar{x}_{t} \mid t \varepsilon T_{U}\right]$ has only a finite number of linearly independent elements, then the set of continuous linear operations on $X$ is just the linear extension of $\left[\bar{x}_{t} \mid t \varepsilon T_{U}, U \varepsilon V\right]$.

Since an $n$-dimensional normed vector space has an $n$-dimensional adjoint space, this is an immediate consequence of Theorems 1.2 and 1.3.

If a weak topology is defined on a linear vector space by means of all finite subsets of a certain class $X^{*}$ of linear operations on the space, then, by the corollary, the set of all linear continuous operations on this space is exactly the linear extension of $X^{*}$.

2. The integral and unconditional summability. Let $S$ be an abstract set possessing a sigma-field $\delta$ of "measurable" subsets $\sigma$. We will study the integration of multiple-valued set functions $x(\sigma)$ having the property that the set $x\left(\sigma_{1}\right)$ contains the set $x\left(\sigma_{2}\right)$ if $\sigma_{1} \supset \sigma_{2}$. A point function $x(s)$ on $S$ to $X$ defines a set function of this type $x(\sigma)$ where $x(\sigma)$ denotes the set of all elements $x(s)$ for $s \varepsilon \sigma$. The function $\alpha(\sigma)$ will be a single-valued, nonnegative, bounded, completely additive measure function on $\delta$. We will designate by $\Delta$ a subdivision of $S$ into a finite or denumerable set of sets $\left(\sigma_{i}\right)$ such that 
$\sigma_{i} \varepsilon \mathcal{S}, \sigma_{i} \cdot \sigma_{j}=0$ if $i \neq j$, and $\sum \sigma_{i}=S$. The expression $\Delta_{1} \geqq \Delta_{2}$ will mean that every set $\sigma^{1}$ of $\Delta_{1}$ is a subset of some $\sigma^{2}$ of $\Delta_{2}$, and $\Delta_{1} \cdot \Delta_{2}$ will be the subdivision of sets $\left(\sigma_{i}{ }^{1} \cdot \sigma_{j}{ }^{2}\right), \sigma^{i}$ of $\Delta_{i}$. The convex extension of the subset $B$ of $X$ which is the set of all $\sum_{1}^{n} a_{i} x_{i}$ where $a_{i} \geqq 0, \sum_{1}^{n} a_{i}=1, x_{i} \varepsilon B$, will be written as cvx $(B)$. $\pi$ will be a finite set of positive integers. $\pi_{1} \geqq \pi_{2}, \pi_{1}+\pi_{2}, \pi_{1} \cdot \pi_{2}$, and $\pi_{1}-\pi_{2}$ will denote respectively inclusion, the integers in $\pi_{1}$ or $\pi_{2}$, the integers in $\pi_{1}$ and $\pi_{2}$, the integers in $\pi_{1}$ not in $\pi_{2} . \sum_{\pi} B_{i}$ will be the set of all $\sum_{\pi} x_{i}$ summed over $\pi$ where $x_{i}$ is an element of the subset $B_{i}$ of $X$. Let $\left\{B_{i}\right\}$ be a finite or denumerable sequence of subsets of $X$. We will designate by $\left[\sum_{\pi} B_{i}\right]$ the set of all sums $\sum_{\pi} B_{i}$. In this notation $\left[\sum_{\pi} B_{i}\right]$ will be called unconditionally summable with respect to $U$ if there exists $\pi_{U}$ such that if $\pi_{i} \geqq \pi_{U}(i=1,2)$, then $\left\|\sum_{\pi_{1}} B_{i}-\sum_{\pi_{2}} B_{i}\right\|_{U}<1 ; \dagger$ and unconditionally summable to the value $x \varepsilon X$ with respect to $U$ if there exists $\pi_{U}$ such that if $\pi \geqq \pi_{U}$, then $\left\|\sum_{\pi} B_{i}-x\right\|_{U}<1$. If the $B_{i}$ are real numbers, then unconditional summability of $\left[\sum_{\pi} B_{i}\right]$ is equivalent to the absolute convergence of $\sum_{i=1}^{\infty} B_{i}[9$, p. 63].

We are now in a position to define the $U$-integral. Let $J_{\Delta}(x, \sigma)$ $=\left[\sum_{\pi} x\left(\sigma \cdot \sigma_{i}\right) \alpha\left(\sigma \cdot \sigma_{i}\right)\right]$ where $\Delta=\left(\sigma_{i}\right)$.

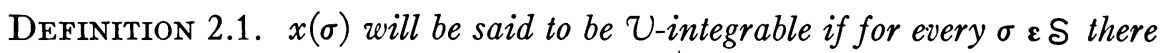
exists a $J(x, \sigma) \varepsilon X$ such that given $U \varepsilon \cup$ there exists $\Delta_{U \sigma}$ for which $J_{\Delta U \sigma}(x, \sigma)$ is unconditionally summable to the value $J(x, \sigma)$ with respect to $U$. We define $J(x, \sigma)$ to be the value of the integral on the set $\sigma$; that is, $J(x, \sigma)=\int_{\sigma} x d \alpha$.

The uniqueness of $J(x, \sigma)$ will be a consequence of Theorem 2.3 to follow.

The essential difference exhibited by this definition is that it does not require the sums $\sum_{1}^{\infty} x_{i} \alpha\left(\sigma_{i}\right)$ where $x_{i} \varepsilon x\left(\sigma_{i}\right)$ to converge. Instead it requires the sums of a subdivision corresponding to a given neighborhood $U$ to be contained in $J(x, \sigma)+U$ whenever the set of integers over which the sum is taken includes a certain finite set. We are thus freed from convergence problems. On the other hand to have demanded the unconditional convergence of the sums $\sum x\left(\sigma_{i}\right) \alpha\left(\sigma_{i}\right)$ would have placed unnecessary restrictions either on $x(\sigma)$ or on $X$. Finally it has been necessary to postulate the existence of $J(x, \sigma)$ because a linear topological space need not be complete.

Theorem 2.2. If, for a given $\Delta, J_{\Delta}(x, S)$ is unconditionally summable with respect to $U$, then $J_{\Delta}(x, \sigma)$ is unconditionally summable with respect to $3 U$ uniformly in $\sigma$ (that is, $\pi_{3 U}$ is the same for all $\sigma$ ).

By hypothesis there exists $\pi_{U}$ such that if $\pi_{i} \geqq \pi_{U}(i=1,2)$, then $\left[\sum_{\pi_{1}} x\left(\sigma_{i}\right) \alpha\left(\sigma_{i}\right)-\sum_{\pi_{2}} x\left(\sigma_{i}\right) \alpha\left(\sigma_{i}\right)\right] \subset U$. Since $U$ is convex, we may write the relationship

† If $B$ is a subset of $X$, then $\|B\|_{U}<1$ means that $B \subset U$. 


$$
\begin{aligned}
U \supset \frac{\alpha\left(\sigma \cdot \sigma_{i_{1}}\right)}{\alpha\left(\sigma_{i_{1}}\right)}\left[\sum _ { \pi _ { U } } \left[x\left(\sigma_{i}\right) \alpha\left(\sigma_{i}\right)\right.\right. & \left.\left.-x\left(\sigma_{i}\right) \alpha\left(\sigma_{i}\right)\right]\right] \\
+ & {\left[1-\frac{\alpha\left(\sigma \cdot \sigma_{i_{1}}\right)}{\alpha\left(\sigma_{i_{1}}\right)}\right]\left[\sum_{\pi_{U}}\left[x\left(\sigma_{i}\right) \alpha\left(\sigma_{i}\right)-x\left(\sigma_{i}\right) \alpha\left(\sigma_{i}\right)\right]\right] }
\end{aligned}
$$

where $i_{1} \varepsilon \pi_{U}$. Let us take any element of $\left[x\left(\sigma_{i}\right) \alpha\left(\sigma_{i}\right)-x\left(\sigma_{i}\right) \alpha\left(\sigma_{i}\right)\right]$ in the first term on the right and take the same element of $\left[x\left(\sigma_{i}\right) \alpha\left(\sigma_{i}\right)-x\left(\sigma_{i}\right) \alpha\left(\sigma_{i}\right)\right]$ in the second except in the case of $i_{1}$ where we always take $\theta \varepsilon\left[x\left(\sigma_{i_{1}}\right) \alpha\left(\sigma_{i_{1}}\right)\right.$ $\left.-x\left(\sigma_{i_{1}}\right) \alpha\left(\sigma_{i_{1}}\right)\right]$ in the second term. This will give

$$
U \supset \sum_{\pi_{U}-i_{1}}\left[x\left(\sigma_{i}\right) \alpha\left(\sigma_{i}\right)-x\left(\sigma_{i}\right) \alpha\left(\sigma_{i}\right)\right]+\left[x\left(\sigma_{i_{1}}\right) \alpha\left(\sigma \cdot \sigma_{i_{1}}\right)-x\left(\sigma_{i_{1}}\right) \alpha\left(\sigma \cdot \sigma_{i_{1}}\right)\right] .
$$

In a like manner we obtain

$$
U \supset \sum_{\pi_{V}-i_{1}-i_{2}}\left[x\left(\sigma_{i}\right) \alpha\left(\sigma_{i}\right)-x\left(\sigma_{i}\right) \alpha\left(\sigma_{i}\right)\right]+\sum_{i_{1}+i_{2}}\left[x\left(\sigma_{i}\right) \alpha\left(\sigma \cdot \sigma_{i}\right)-x\left(\sigma_{i}\right) \alpha\left(\sigma \cdot \sigma_{i}\right)\right]
$$

where $i_{2} \varepsilon \pi_{U}$. Treating successively the integers of $\pi_{U}$, we will finally arrive at (1) $U \supset \sum_{\pi_{U}}\left[x\left(\sigma_{i}\right) \alpha\left(\sigma \cdot \sigma_{i}\right)-x\left(\sigma_{i}\right) \alpha\left(\sigma \cdot \sigma_{i}\right)\right] \supset \sum_{\pi_{U}}\left[x\left(\sigma \cdot \sigma_{i}\right) \alpha\left(\sigma \cdot \sigma_{i}\right)-x\left(\sigma \cdot \sigma_{i}\right) \alpha\left(\sigma \cdot \sigma_{i}\right)\right]$. If $\pi_{0} \cdot \pi_{U}=0$, then

$$
\begin{aligned}
\sum_{\pi_{0}} x\left(\sigma \cdot \sigma_{i}\right) \alpha\left(\sigma \cdot \sigma_{i}\right) & \subset \sum_{\pi_{0}} x\left(\sigma_{i}\right) \alpha\left(\sigma \cdot \sigma_{i}\right) \subset \sum_{\pi_{0}} \operatorname{cvx}\left[x\left(\sigma_{i}\right) \alpha\left(\sigma_{i}\right)+\theta\right] \\
\subset \operatorname{cvx} & {\left[\sum_{\pi} x\left(\sigma_{i}\right) \alpha\left(\sigma_{i}\right) \mid \text { finite set of } \pi \text { 's where } \pi \cdot \pi_{U}=0\right] \subset U . }
\end{aligned}
$$

(1) and (2) together give $\left(\sum_{\pi_{1}} x\left(\sigma \cdot \sigma_{i}\right) \alpha\left(\sigma \cdot \sigma_{i}\right)-\sum_{\pi_{2}} x\left(\sigma \cdot \sigma_{i}\right) \alpha\left(\sigma \cdot \sigma_{i}\right)\right) \subset 3 U$ for all $\sigma \varepsilon S$ where $\pi_{i} \geqq \pi_{U}(i=1,2)$.

THEOREM 2.3. If $J_{\Delta_{0}}(x, \sigma)$ is unconditionally summable to $J(x, \sigma)$ with respect to $U$, then for any $\Delta \geqq \Delta_{0}, J_{\Delta}(x, \sigma)$ is unconditionally summable to $J(x, \sigma)$ with respect to $4 U$.

Without loss of generality we may consider only the case $\sigma=S$. By hypothesis there exists $\pi_{0}$ such that if $\pi \geqq \pi_{0}$, then $\left(\sum_{\pi} x\left(\sigma_{i}\right) \alpha\left(\sigma_{i}\right)-J(x, S)\right) \subset U$ where $\Delta_{0}=\left(\sigma_{i}\right)$. Let $\left(\sigma_{j}{ }^{i}\right)$ be a subdivision of $\sigma_{i}$. Then $\Delta=\left(\sigma_{j}{ }^{i}\right)(i, j=1,2, \cdots)$ is a subdivision of $S$. We first propose to prove a lemma.

Leмma. Let $x\left(\sigma_{i}\right) \alpha\left(\sigma_{i}\right)+Y_{i} \subset b_{i} U$ where $Y_{i}$ is a subset of $X$ and $b_{i}>0$. Then there exists an $n_{i}$ such that if $n \geqq n_{i}$ then

and

$$
\left\|\left[\sum_{j=n+1}^{\infty} \alpha\left(\sigma_{j}^{i}\right)\right] x\left(\sigma_{i}\right)\right\|_{U}<\frac{1}{2^{i}}
$$

$$
\sum_{j=1}^{n} x\left(\sigma_{j}^{i}\right) \alpha\left(\sigma_{j}^{i}\right)+Y_{i} \subset b_{i} U+\frac{1}{2^{i}} U .
$$


Since $U$ is convex,

$$
b_{i} U \supset \operatorname{cvx}\left[x\left(\sigma_{i}\right) \alpha\left(\sigma_{i}\right)+Y_{i}\right] \supset \frac{1}{c_{n}^{i}} \sum_{j=1}^{n} x\left(\sigma_{j}^{i}\right) \alpha\left(\sigma_{j}^{i}\right)+Y_{i}
$$

where $c_{n}{ }^{i}=\sum_{j=1} \alpha\left(\sigma_{j}{ }^{i}\right) / \alpha\left(\sigma_{i}\right) \leqq 1$. Hence

$$
\sum_{j=1}^{n} x\left(\sigma_{j}^{i}\right) \alpha\left(\sigma_{j}^{i}\right)+c_{n}^{i} Y_{i} \subset c_{n}^{i} b_{i} U_{i} \subset b_{i} U
$$

As $\left\|x\left(\sigma_{i}\right) \alpha\left(\sigma_{i}\right)+Y_{i}\right\|_{U}<b_{i}$, there exists an $M_{i}$ for which $\left\|x\left(\sigma_{i}\right) \alpha\left(\sigma_{i}\right)\right\|_{U}$ $+\left\|Y_{i}\right\|_{U}<M_{i}$. Now $c_{n}{ }^{i} \rightarrow 1$ as $n \rightarrow \infty$ so that there will be an $n_{i}$ such that if $n \geqq n_{i}$ then $\left\|\left(1-c_{n}{ }^{i}\right) Y_{i}\right\|_{U}<1 / 2^{i}$ and $\left\|\left(1-c_{n}{ }^{i}\right) x\left(\sigma_{i}\right) \alpha\left(\sigma_{i}\right)\right\|_{U}<1 / 2^{i}$. Therefore $\sum_{j=1}^{n} x\left(\sigma_{j}^{i}\right) \alpha\left(\sigma_{j}{ }^{i}\right)+Y_{i}<b_{i} U+U / 2^{i}$ for $n \geqq n_{i}$.

We will now apply this lemma successively to the elements of $\pi_{0}$. If $i_{n}$ is the $n$th integer of $\pi_{0}$ so chosen, then

$$
Y_{i_{n}}=\sum_{\pi_{0}-\pi_{n}-i_{n}} x\left(\sigma_{i}\right) \alpha\left(\sigma_{i}\right)+\sum_{\pi_{n}}\left[\sum_{j=1}^{n_{i}} x\left(\sigma_{j}^{i}\right) \alpha\left(\sigma_{j}{ }^{i}\right)\right]-J(x, S),
$$

and $b_{i_{n}}=\sum_{0+\pi_{n}} 1 / 2^{i}$ where $\pi_{n}=\left(i_{1}, i_{2}, \cdots, i_{n-1}\right)$. We finally obtain

$$
\left\{\sum_{\pi_{0}}\left[\sum_{j=1}^{n_{i}} x\left(\sigma_{i}^{j}\right) \alpha\left(\sigma_{i}^{j}\right)\right]-J(x, S)\right\} \subset\left(\sum_{0+\pi_{0}} \frac{1}{2^{i}}\right) U \subset 2 U .
$$

Let $\nu$ be a finite set of number pairs $(i, j)$ which designate the superscript and subscript respectively of $\sigma_{j}{ }^{i}$. Define $\nu^{\prime}=\left[(i, j) \mid i \varepsilon \pi_{0}, j=1, \cdots, n_{i}\right]$. If $\nu \geqq \nu^{\prime}$, then the terms of $\sum_{\nu} x\left(\sigma_{j}{ }^{i}\right) \alpha\left(\sigma_{j}{ }^{i}\right)$ not included in the above sum may be split into two partial sums $\Sigma^{\prime}$ and $\Sigma^{\prime \prime}$ where $\Sigma^{\prime}$ contains only terms from $x\left(\sigma_{i}\right)$, $i \notin \pi_{0}$, and $\Sigma^{\prime \prime}$ contains only terms from $x\left(\sigma_{i}\right), i \varepsilon \pi_{0}$. There exists $\pi_{1}\left(\pi_{1} \cdot \pi_{0}=0\right)$ such that

$$
\begin{aligned}
& \Sigma^{\prime} \subset \sum_{i \neq \pi_{0}, i \varepsilon \pi_{1}} \operatorname{cvx}\left[x\left(\sigma_{i}\right) \alpha\left(\sigma_{i}\right)+\theta\right] \\
& \quad \subset \operatorname{cvx}\left[\sum_{\pi} x\left(\sigma_{i}\right) \alpha\left(\sigma_{i}\right) \mid \text { finite set of } \pi \text { 's where } \pi \cdot \pi_{0}=0\right] \subset U .
\end{aligned}
$$

Also

$$
\Sigma^{\prime \prime} \subset \sum_{i \varepsilon x_{0},(i, j) \text { of } \Sigma^{\prime \prime}} x\left(\sigma_{a_{j}{ }^{i}}\right) \alpha\left(\sigma_{a_{j}}{ }^{i}\right) \subset \sum_{\pi_{0}}\left[\sum_{(i, j) \text { of } \Sigma^{\prime \prime}} x\left(\sigma_{a_{j}{ }^{i}}\right) \alpha\left(\sigma_{a_{j}}{ }^{i}\right)\right] .
$$

Now in the $(i, j)$ of $\Sigma^{\prime \prime}$ we have $j>n_{i}$ so that $\left[\sum_{(i, j) \text { of } \Sigma^{\prime \prime}} \alpha\left(\sigma_{a_{j}}{ }^{i}\right)\right] x\left(\sigma_{i}\right) \subset U / 2^{i}$. 


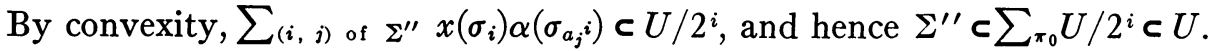
Finally if $\nu \geqq \nu^{\prime}$,

$$
\sum_{\nu} x\left(\sigma_{j}^{i}\right) \alpha\left(\sigma_{j}^{i}\right)-J(x, S) \subset 2 U+U+U \subset 4 U .
$$

CoRollary 2.4. If $J_{\Delta_{1}}(x, \sigma)$ and $J_{\Delta_{2}}(x, \sigma)$ are unconditionally summable to $J(x, \sigma)$ with respect to $U_{1}$ and $U_{2}$ respectively, then $J_{\Delta_{1} \cdot \Delta_{2}}(x, \sigma)$ is unconditionally summable to $J(x, \sigma)$ with respect to both $4 U_{1}$ and $4 U_{2}$.

3. Basic properties of the integral. A set function $y(\sigma)$ on $S$ to $X$ will be said to be absolutely continuous if for every $U \varepsilon V$ there exists a $\delta_{U}>0$ such that if $\alpha(\sigma)<\delta_{U}$ then $y(\sigma) \subset U$. Absolute continuity is clearly relative to $\alpha(\sigma)$.

Theorem 3.1. If $x(\sigma)$ is $U$-integrable, then $\int_{\sigma} x d \alpha$ is absolutely continuous.

Since $x(\sigma)$ is $U$-integrable, it follows from Theorem 2.2 that to each $U \varepsilon V$ there corresponds a $\Delta_{U}$ and $\pi_{U}$ such that $\left\|\sum_{\pi_{U}} x\left(\sigma \cdot \sigma_{i}\right) \alpha\left(\sigma \cdot \sigma_{i}\right)-J(x, \sigma)\right\|_{U}<1 / 2$ for all $\sigma \varepsilon S$. As $\left\|x\left(\sigma_{i}\right)\right\|_{U}<\infty$ there exists a $\delta_{U}>0$ for which if $\alpha(\sigma)<\delta_{U}$ then $\left\|x\left(\sigma_{i}\right) \alpha(\sigma)\right\|_{U}<1 / 2^{i+1}\left(i \varepsilon \varepsilon \pi_{U}\right)$. But then $\left\|\sum_{\pi U} x\left(\sigma \cdot \sigma_{i}\right) \alpha\left(\sigma \cdot \sigma_{i}\right)\right\|_{U}<1 / 2$ and hence if only $\alpha(\sigma)<\delta_{U}$,

$$
\|J(x, \sigma)\|_{U} \leqq\left\|\sum_{\pi_{U}} x\left(\sigma \cdot \sigma_{i}\right) \alpha\left(\sigma \cdot \sigma_{i}\right)-J(x, \sigma)\right\|_{U}+\left\|\sum_{x_{U}} x\left(\sigma \cdot \sigma_{i}\right) \alpha\left(\sigma \cdot \sigma_{i}\right)\right\|_{U}<1 .
$$

THeOREm 3.2. If $x(\sigma)$ is U-integrable, then $\int_{\sigma} x d \alpha$ is additive.

It follows from Theorem 2.2 that to each $U \varepsilon V$ there corresponds a $\Delta_{U}=\left(\sigma_{i}\right)$ and $\pi_{U}$ such that if $\pi \geqq \pi_{U}$, then $\left\|\sum_{\pi} x\left(\sigma \cdot \sigma_{i}\right) \alpha\left(\sigma \cdot \sigma_{i}\right)-J(x, \sigma)\right\|_{U}<1 / 10$ for all $\sigma \varepsilon S$. Suppose $\sigma^{0}=\sigma^{1}+\sigma^{2}$ and $\sigma^{1} \cdot \sigma^{2}=0$. The subdivision $\left(\sigma_{i}{ }^{0}\right)$ $=\left\{\left(\sigma^{1} \cdot \sigma_{j}\right),\left(\sigma^{2} \cdot \sigma_{j}\right)\right\}$ of $\sigma^{0}$ is greater than $(\geqq)$ the subdivision $\left(\sigma^{0} \cdot \sigma_{i}\right)$ of $\sigma^{0}$. Hence by Theorem 2.3 there exists $\pi_{0}$ such that if $\pi \geqq \pi_{0}$ then

$$
\left\|\sum_{\pi} x\left(\sigma_{i}{ }^{0}\right) \alpha\left(\sigma_{i}{ }^{0}\right)-J\left(x, \sigma^{0}\right)\right\|_{U}<2 / 5 .
$$

When we combine these statements, taking $\pi \geqq \pi_{0}$ sufficiently large so that among the sets $\sigma_{i}{ }^{0}(i \varepsilon \pi)$ are the sets $\left(\sigma^{1} \cdot \sigma_{i}\right)$ and $\left(\sigma^{2} \cdot \sigma_{i}\right)$ for $i \varepsilon \pi_{U}$, we obtain

$$
\begin{aligned}
&\left\|J\left(x, \sigma^{1}\right)+J\left(x, \sigma^{2}\right)-J\left(x, \sigma^{0}\right)\right\|_{U} \\
& \leqq \sum_{k=0}^{2}\left\|J\left(x, \sigma^{k}\right)-\sum_{\pi} x\left(\sigma^{k} \cdot \sigma_{i}{ }^{0}\right) \alpha\left(\sigma^{k} \cdot \sigma_{i}{ }^{0}\right)\right\|_{U} \\
&+\sum_{k=1}^{2}\left\|\sum_{x}\left[x\left(\sigma^{k} \cdot \sigma_{i}{ }^{0}\right) \alpha\left(\sigma^{k} \cdot \sigma_{i}{ }^{0}\right)-x\left(\sigma^{k} \cdot \sigma_{i}{ }^{0}\right) \alpha\left(\sigma^{k} \cdot \sigma_{i}{ }^{0}\right)\right]\right\|_{U}<1 .
\end{aligned}
$$

Therefore $J\left(x, \sigma^{0}\right)=J\left(x, \sigma^{1}\right)+J\left(x, \sigma^{2}\right)$. 
Theorem 3.3. If $x(\sigma)$ is $U$-integrable, then $\int_{\sigma} x d \alpha$ is completely additive and $\sum_{i} \int_{\sigma_{i}} x d \alpha$ is unconditionally convergent.

Let $\left\{\sigma_{i}\right\}$ be a sequence of disjoint sets of $S$. Since $\sum \alpha\left(\sigma_{i}\right)<\infty$, Theorems 3.1 and 3.2 imply $\lim _{n}\left\|\int_{\Sigma \sigma_{i}} x d \alpha-\sum_{i=1}^{n} \int_{\sigma_{i}} x d \alpha\right\|_{U}=0$. As the same considerations hold for any subsequence of $\left\{\sigma_{i}\right\}$, the sum is by definition unconditionally convergent.

TheOREM 3.4. If $x(\sigma)$ and $y(\sigma)$ are $U$-integrable and $m$ is a real number, then $m \cdot x(\sigma)$ and $x(\sigma)+y(\sigma)$ are $U$-integrable, $m J(x, \sigma)=J(m x, \sigma)$, and $J(x+y, \sigma)=J(x, \sigma)+J(y, \sigma)$.

The conclusion about $m \cdot x(\sigma)$ follows from the fact that when $J_{\Delta}(x, \sigma)$ is unconditionally summable with respect to $U$ to the value $J(x, \sigma)$, then $J_{\Delta}(m x, \sigma)$ is unconditionally summable to $m J(x, \sigma)$ with respect to $m U$. Finally if $J_{\Delta_{1}}(x, \sigma)$ and $J_{\Delta_{2}}(x, \sigma)$ are unconditionally summable with respect to $U$ to the values $J(x, \sigma)$ and $J(y, \sigma)$ respectively, then $J_{\Delta}(x+y, \sigma)$ where $\Delta \geqq \Delta_{1} \cdot \Delta_{2}$ is unconditionally summable to $J(x, \sigma)+J(y, \sigma)$ with respect to $8 U$ by Theorem 2.3 .

4. The integral in a complete space. The conditions for integrability can be relaxed when $X$ satisfies a certain type of completeness property. It is convenient to define this property by means of the general limit notion of E. H. Moore and H. L. Smith $[10$, p. 103] on a class $D$ with a relation $\mathrm{R}$ on $D D$ which is transitive and compositive. Each element of $D$ will represent a unique subdivision $\Delta$ of $S$ and neighborhood $U \varepsilon V$, and will be designated by $\Delta U$. We define $\Delta_{1} U_{1}$ R $\Delta_{2} U_{2}$ to mean $\Delta_{1} \geqq \Delta_{2}$ and $U_{1} \subset U_{2}$. A sequence $x_{n} \varepsilon X$ will be called a fundamental sequence if for each $U \varepsilon \mathcal{V}$ there is an $N_{U}$ such that if $m, n \geqq N_{U}$ then $\left(x_{n}-x_{m}\right) \varepsilon U . X$ will be said to be sequentially complete if every fundamental sequence is convergent. $X$ will be said to be complete with respect to $D$ if every "fundamental $D$-sequence," $x(\Delta U) \subset X$ (that is, every sequence such that for each $U \varepsilon \cup$ there is a $\Delta U$ such that $\Delta_{i} U_{i} \mathrm{R} \Delta U$ $(i=1,2)$ implies $\left.\left[x\left(\Delta_{1} U_{1}\right)-x\left(\Delta_{2} U_{2}\right)\right] \varepsilon U\right)$ is "convergent" (that is, an $x \varepsilon X$ exists such that for each $U \varepsilon V$ there is a $\Delta U$ such that $\Delta_{1} U_{1} \mathrm{R} \Delta U$ implies $\left.\left[x\left(\Delta_{1} U_{1}\right)-x\right] \varepsilon U\right)$.

TheOREM 4.1. If $X$ is complete with respect to $D$ and $x(\sigma)$ has the property that for every $U \varepsilon \cup$ there exists a $\Delta_{U}$ such that $J_{\Delta_{U}}(x, S)$ is unconditionally summable with respect to $U$, then $x(\sigma)$ is $U$-integrable.

By hypothesis there exists $\Delta_{U}$ and $\pi_{U}$ such that if $\pi_{i} \geqq \pi_{U}$ then

$$
\left\|\sum_{\pi_{1}} x\left(\sigma_{i}\right) \alpha\left(\sigma_{i}\right)-\sum_{\pi_{2}} x\left(\sigma_{i}\right) \alpha\left(\sigma_{i}\right)\right\|_{U}<1 .
$$


Let us designate by $\mathcal{F}_{\Delta U}(x, \sigma)$ some one element of the set $\sum_{\pi_{U}} x\left(\sigma \cdot \sigma_{i}\right) \alpha\left(\sigma \cdot \sigma_{i}\right)$. Theorem 2.2 implies that $\left\|\sum_{\pi} x\left(\sigma \cdot \sigma_{i}\right) \alpha\left(\sigma \cdot \sigma_{i}\right)-\mathcal{F}_{\Delta U}(x, \sigma)\right\|_{U}<3$ for all $\sigma \varepsilon \mathcal{S}$ if $\pi \geqq \pi_{U}$. By Theorem 2.3 , if $\Delta_{j}=\left(\sigma_{i}{ }^{j}\right) \geqq \Delta_{U}$, then there exists $\pi_{j}$ such that for $\pi \geqq \pi_{j},\left\|\sum_{\pi} x\left(\sigma \cdot \sigma_{i}{ }^{j}\right) \alpha\left(\sigma \cdot \sigma_{i}{ }^{j}\right)-\mathcal{F}_{\Delta U}(x, \sigma)\right\|_{U}<12$. Finally when $\Delta_{j} U_{j} \mathrm{R} \Delta U$, we have

$$
\begin{aligned}
\left\|\sum_{\pi} x\left(\sigma \cdot \sigma_{i}^{j}\right) \alpha\left(\sigma \cdot \sigma_{i}^{j}\right)-\mathcal{F}_{\Delta_{j} U_{j}}(x, \sigma)\right\|_{U} & \\
\leqq & \left\|\sum_{\pi} x\left(\sigma \cdot \sigma_{i}^{j}\right) \alpha\left(\sigma \cdot \sigma_{i}^{j}\right)-\mathcal{F}_{\Delta, U_{j}}(x, \sigma)\right\|_{U_{j}}<1
\end{aligned}
$$

if only $\pi \geqq \pi_{U_{j}}$. Combining these statements gives the result that for $\Delta_{j} U_{j} \mathrm{R} \Delta U(j=1,2)$,

$$
\left\|\mathcal{F}_{\Delta, U_{1}}(x, \sigma)-\mathcal{F}_{\Delta_{2} U_{2}}(x, \sigma)\right\|_{U} \leqq \sum_{j=1}^{2}\left\|\mathcal{F}_{\Delta_{j} U_{j}}(x, \sigma)+\mathcal{F}_{\Delta U}(x, \sigma)\right\|_{U}<2 \cdot 13=26 .
$$

Completeness with respect to $D$ then implies the existence of $J(x, \sigma)$ for which if $U \varepsilon \cup$ there exists a $\Delta_{U}$ and $\pi_{U}$ such that if $\pi \geqq \pi_{U}$, then

$$
\left\|J(x, \sigma)-\sum_{\pi} x\left(\sigma \cdot \sigma_{i}\right) \alpha\left(\sigma \cdot \sigma_{i}\right)\right\|_{U}<1+\left\|\mathcal{F}_{\Delta U}(x, \sigma)-J(x, \sigma)\right\|_{U}<1+26=27 .
$$

LEMMA 4.2. If $X$ is sequentially complete and satisfies the first countability axiom, then $X$ is complete with respect to $D$.

Let $\left\{U_{n}\right\}$ where $U_{n} \supset U_{n+1}$ be a complete neighborhood system of $\theta$. If $x(\Delta U)$ is a fundamental $D$-sequence, then for each $U \varepsilon V$ there exists a $\Delta_{U} U \varepsilon D$ such that $\Delta_{i} U_{i} \mathrm{R} \Delta_{U} U(i=1,2)$ implies $\left\|x\left(\Delta_{1} U_{1}\right)-x\left(\Delta_{2} U_{2}\right)\right\|_{U}<1$. If we define $\Delta_{n}=\prod_{i=1}^{n} \Delta_{U_{i}}$, then $k_{i} \geqq n$ implies $\Delta_{k_{i}} U_{k_{i}} \mathrm{R} \Delta_{U_{k_{i}}} U_{k_{i}} \mathrm{R} \Delta_{U_{n}} U_{n}$ and $\left\|x\left(\Delta_{k_{1}} U_{k_{1}}\right)-x\left(\Delta_{k_{2}} U_{k_{2}}\right)\right\|_{U_{n}}<1$. As $X$ is sequentially complete, there exists $x_{0}=\lim _{n} x\left(\Delta_{n} U_{n}\right)$. Now given $U \varepsilon V$, there exists $U_{n_{0}} \subset U$. Let $\Delta^{\prime}=\Delta_{U} \cdot \Delta_{n_{0}}$. Then

$$
\begin{aligned}
\left\|x_{0}-x\left(\Delta_{U} U\right)\right\|_{U} \leqq & \left\|x_{0}-x\left(\Delta_{n_{0}} U_{n_{0}}\right)\right\|_{U}+\left\|x\left(\Delta_{n_{0}} U_{n_{0}}\right)-x\left(\Delta^{\prime} U_{n_{0}}\right)\right\|_{U_{n}} \\
& +\left\|x\left(\Delta^{\prime} U_{n_{0}}\right)-x\left(\Delta_{U} U\right)\right\|_{U} \leqq 3
\end{aligned}
$$

COROLLARY 4.3. If $X$ is sequentially complete and satisfies the first countability axiom, and if $x(\sigma)$ has the property that for every $U \varepsilon \cup$ there exists $a \Delta_{U}$ such that $J_{\Delta_{U}}(x, S)$ is unconditionally summable with respect to $U$, then $x(\sigma)$ is V-integrable.

This is an immediate consequence of Theorem 4.1 and Lemma 4.2. 
5. The continuous transform of an integrable function. Let $X$ and $Y$ be linear topological spaces with the convex neighborhood systems $U$ and $U$ of their respective null elements. The following theorem can then be proved:

THEOREM 5.1. If $T$ is a linear continuous transformation on $X$ to $Y$ and if $x(\sigma)$ is U-integrable, then $T[x(\sigma)]$ is U-integrable and $J(T[x], \sigma)=T[J(x, \sigma)]$.

Since $T$ is continuous, for every $V \varepsilon \cup$ there exists a $U \varepsilon V$ such that $T(U) \subset V$. The $U$-integrability of $x(\sigma)$ implies the existence of $J(x, \sigma)$, $\Delta_{U}, \pi_{U}$ such that if $\pi \geqq \pi_{U}$ then $\sum_{\pi} x\left(\sigma \cdot \sigma_{i}\right) \alpha\left(\sigma \cdot \sigma_{i}\right)-J(x, \sigma) \subset U$. Hence for $\pi \geqq \pi_{U}$ we have $\sum_{\pi} T\left[x\left(\sigma \cdot \sigma_{i}\right)\right] \alpha\left(\sigma \cdot \sigma_{i}\right)-T[J(x, \sigma)] \subset V$. It follows that $T[x(\sigma)]$ is $U$-integrable and that $J(T[x], \sigma)=T[J(x, \sigma)]$.

CoROllary 5.2. If $\bar{x}$ is a linear continuous operation on $X$ and if $x(\sigma)$ is $U$-integrable, then $\bar{x}[x(\sigma)]$ is Lebesgue integrable and $\bar{x}[J(x, \sigma)]=\int_{\sigma} \bar{x}[x] d \alpha$.

In this case $Y$ is the space of real numbers. For real-valued functions our integral reduces to Fréchet's interpretation of the Lebesgue integral (11). The corollary then follows from Theorem 5.1.

If $x(s)$ is a multiple-valued point function on $S$ to $X$ and if the set function $x(\sigma) \equiv[x(s) \mid s \varepsilon \sigma]$ is $U$-integrable to the value $J(x, \sigma)$, then we will say that $x(s)$ is $U$-integrable to the value $J(x, \sigma)$. Further a set of linear continuous operations $\mathrm{I}$ on $X$ will be said to be total if when $\bar{x}(x)=0$ for all $\bar{x} \varepsilon \Gamma$, then $x=\theta[6, \mathrm{p} .42]$.

THEOREM 5.3. If the set of linear continuous operations on $X$ contains a denumerable subset which is total and if $x(s)$ and $y(s)$ are integrable to the same values on $\mathrm{S}$, then $x(s)=y(s)$ a.e.

Let $\left\{\bar{x}_{n}\right\}$ be a total denumerable subset of linear continuous operations on $X$. Then for all $\sigma \varepsilon S, \int_{s} \bar{x}_{n}[x(s)-y(s)] d \alpha=0$ by Corollary 5.2. Hence $\bar{x}_{n}[x(s)-y(s)]=0$ on $S-\sigma_{n}$ where $\alpha\left(\sigma_{n}\right)=0$. Therefore $\bar{x}_{n}[x(s)-y(s)]=0$ on $S-\sigma_{0}=S-\sum \sigma_{i}$ for all $n$, and $\alpha\left(\sigma_{0}\right)=0$. As $\left\{\bar{x}_{n}\right\}$ is total, $x(s)=y(s)$ on $S-\sigma_{0}$, which proves the theorem.

The conjugate space $\bar{X}$ to a Banach space $X$ has been defined by Dunford to be a fundamentally separable space with a determining manifold $\Gamma$ in case $\Gamma$ is a separable linear manifold in $\bar{X}$ such that for every $x \varepsilon X$, we have l.u.b. $[|\bar{x}(x)| \mid \bar{x} \varepsilon \Gamma,\|\bar{x}\|=1]=\|x\|[2$, p. 310]. It is clear that if $X$ is a Banach space with its weak neighborhood topology or even its norm topology (see $\$ 1$ ) and $\bar{X}$ is a fundamentally separable space, then the set of linear continuous operations on $X$ contains a denumerable subset which is total. Theorem 5.3 is therefore applicable to such spaces.

6. Convergence of integrable functions. We prove first the following theorem. 
Theorem 6.1. If $x_{n}(\sigma)$ are U-integrable and if, for all $\sigma \varepsilon S,\left\{\int_{\sigma} x_{n} d \alpha\right\}$ is a fundamental sequence, then the integrals are equi-absolutely continuous.

The argument of Saks (12) is applicable here. With the distance function $\left(\sigma_{1}, \sigma_{2}\right)=\alpha\left(\sigma_{1}+\sigma_{2}-\sigma_{1} \cdot \sigma_{2}\right)$ the family of sets $S$ becomes a complete metric space. Let $F_{n}(\sigma)=\int_{\sigma} x_{n} d \alpha$ and $\mathcal{S}_{q} \equiv\left[\sigma \varepsilon \delta\right.$ such that $\left.\left\|F_{n}(\sigma)-F_{m}(\sigma)\right\|_{U} \leqq e / 3, m, n \geqq q\right]$. Since $F_{n}(\sigma)$ is absolutely continuous by Theorem 3.1 , the sets $S_{q}$ are closed. Also $S=\sum_{1}^{\infty} S_{q}$. Hence by the Baire category theorem there exists an integer $q_{0}$ and a sphere $K\left(\sigma_{0}, r\right) \subset \mathcal{S}_{q_{0}}$. Let $\delta<r$ be such that $\left\|F_{q_{0}}(\sigma)\right\|_{U} \leqq e / 3$ if $\alpha(\sigma)<\delta$. Then for all $\sigma$ such that $\alpha(\sigma)<\delta<r, \sigma_{1}=\sigma+\left(\sigma_{0}-\sigma\right)$ and $\sigma_{2}=\sigma_{0}-\sigma$ are in $K\left(\sigma_{0}, r\right)$ so that for $m, n \geqq q_{0}$,

$$
\left\|F_{n}(\sigma)-F_{m}(\sigma)\right\|_{U} \leqq\left\|F_{n}\left(\sigma_{1}\right)-F_{m}\left(\sigma_{1}\right)\right\|_{U}+\left\|F_{n}\left(\sigma_{2}\right)-F_{m}\left(\sigma_{2}\right)\right\|_{U} \leqq 2 e / 3 .
$$

Therefore $\left\|F_{n}(\sigma)\right\|_{U} \leqq e$ if $\alpha(\sigma)<\delta$ and $n \geqq q_{0}$.

The integrable functions dealt with in the remainder of the paper will be single-valued point functions on $S$ to $X$. As previously stated the integrability of $x(s)$ depends directly upon the integrability of the set function $x(\sigma) \equiv[x(s) \mid s \varepsilon \sigma]$, and the integral of $x(s)$ is defined to be that of $x(\sigma)$.

We would like to extend to $X$ the notion of approximate convergence introduced by Dunford [2, p. 343]. Let $\sigma(n, e, U) \equiv\left[s \mid\left\|x_{n}(s)-x(s)\right\|_{U} \geqq e\right]$. The sequence $x_{n}(s)$ will be said to approach $x(s)$ approximately on $S$ if for every $n, U$, and $e>0$ there is a measurable set $\sigma^{\prime}(n, e, U) \supset \sigma(n, e, U)$ such that $\lim _{n} \alpha\left[\sigma^{\prime}(n, e, U)\right]=0$.

We are now in a position to prove the following result:

TheOREм 6.2. Let $X$ be sequentially complete, let $x_{n}(s)$ be U-integrable, and let $x_{n}(s) \rightarrow x(s)$ approximately. Then the following statements are equivalent:

(1) $x(s)$ is $U$-integrable and

$$
\lim _{n} \int_{\sigma} x_{n} d \alpha=\int_{\sigma} x d \alpha
$$

uniformly in $\sigma$.

(2) $\lim _{n} \int_{\sigma} x_{n} d \alpha$ exists for every $\sigma \varepsilon S$.

(3) $\int_{\sigma} x_{n} d \alpha$ are equi-absolutely continuous.

That (1) implies (2) is obvious, while Theorem 6.1 proves that (2) implies (3). There remains only the proof that (3) implies (1). For this purpose we demonstrate

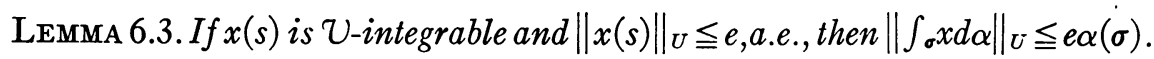

For given $\eta$ and $U$ there exist $\Delta_{\eta U}$ and $\pi_{\eta U}$ such that $\| \sum_{\pi_{\eta} U} x\left(\sigma \cdot \sigma_{i}\right) \alpha\left(\sigma \cdot \sigma_{i}\right)$ $-J(x, \sigma) \|_{U}<\eta$ where $\Delta_{\eta U}=\left(\sigma_{i}\right)$ and $\sigma_{1} \equiv\left[s \mid\|x(s)\|_{U}>e\right]$. But 


$$
\left\|\sum_{\pi_{\eta U}} x\left(\sigma \cdot \sigma_{i}\right) \alpha\left(\sigma \cdot \sigma_{i}\right)\right\|_{U} \leqq \sum_{\pi_{\eta U}}\left\|x\left(\sigma \cdot \sigma_{i}\right)\right\|_{U} \alpha\left(\sigma \cdot \sigma_{i}\right) \leqq e \alpha(\sigma) .
$$

Hence $\|J(x, \sigma)\|_{U} \leqq e \alpha(\sigma)+\eta$ where $\eta$ is arbitrarily small.

To continue the proof of the theorem, we will first show that $\lim _{n} \int_{\sigma} x_{n} d \alpha$ exists uniformly in $\sigma$. By hypothesis, given $U$ and $e>0$ there exists $\delta_{U e}$ such that if $\alpha(\sigma)<\delta_{U_{e}}$, then $\left\|\int_{\sigma} x_{n} d \alpha\right\|_{U}<e$, and further, given $U$ and $\delta>0$ there exists $N_{U \delta}$ such that if $n \geqq N_{U \delta}$ then $\alpha\left[\sigma^{\prime}(n, \delta, U)\right]<\delta$. Let $\delta_{0}$ be the smaller of $e$ and $\delta_{U e}$. Then for $m, n \geqq N_{U \delta_{0}}$ we have $\left\|x_{n}(s)-x_{m}(s)\right\|_{U}<2 \delta_{0}$ on $S-\sigma^{\prime}\left(n, \delta_{0}, U\right)-\sigma^{\prime}\left(m, \delta_{0}, U\right)$. If $\sigma_{n m}=\sigma^{\prime}\left(n, \delta_{0}, U\right)+\sigma^{\prime}\left(m, \delta_{0}, U\right)$, then using Lemma 6.3,

$$
\begin{aligned}
& \left\|\int_{\sigma}\left[x_{n}(s)-x_{m}(s)\right] d \alpha\right\|_{U} \leqq\left\|\int_{\sigma-\sigma_{n m}}\left[x_{n}(s)-x_{m}(s)\right] d \alpha\right\|_{U} \\
& +\left\|\int_{\sigma_{n m}}\left[x_{n}(s)-x_{m}(s)\right] d \alpha\right\|_{U} \leqq 2 \delta_{0} \alpha(S)+2 e \leqq(2 \alpha(S)+2) e .
\end{aligned}
$$

This together with the fact that $X$ is sequentially complete implies that $\lim _{n} \int_{\sigma} x_{n} d \alpha$ exists uniformly in $\sigma$.

Given $U \varepsilon V$, let us select a subsequence, which for notational convenience we will designate as $\left\{x_{n}(s)\right\}$, having the properties: (1) $\left\|\int_{\sigma} x_{n} d \alpha-\lim _{n} \int_{\sigma} x_{n} d \alpha\right\|_{U}$ $<1 / 2^{n}$ for all $\sigma \varepsilon S$, and $(2)\left\|x_{n}(s)-x(s)\right\|_{U}<1 /\left(2^{n} \alpha(S)\right)$ on $S-\tau_{n}$, where $\tau_{n} \varepsilon S$, $\tau_{n} \supset \tau_{n+1}$, and $\alpha\left(\tau_{n}\right)<1 / 2^{n}$. Relation (2) is a consequence of approximate convergence. We will now show that $x(s)$ is $\mathcal{U}$-integrable to the value $J(\sigma)=\lim _{n} \int_{\sigma} x_{n} d \alpha$. As $x_{n}(s)$ is integrable, there exists a $\Delta_{n}$ and a $\pi_{n}$ such that if $\pi \geqq \pi_{n}$ then

$$
\left\|\sum_{\pi} x_{n}\left(\sigma \cdot \sigma_{i}^{n}\right) \alpha\left(\sigma \cdot \sigma_{i}^{n}\right)-J\left(x_{n}, \sigma\right)\right\|_{U}<\frac{1}{2^{n}}
$$

where $\Delta_{n}=\left(\sigma_{i}{ }^{n}\right)$. Hence for $\sigma \subset S-\tau_{n}$ and $\pi \geqq \pi_{n}$,

$$
\begin{aligned}
& \left\|\sum_{x} x\left(\sigma \cdot \sigma_{i}^{n}\right) \cdot \alpha\left(\sigma \cdot \sigma_{i}^{n}\right)-J(\sigma)\right\|_{U} \leqq \text { l.u.b. }_{s_{i \varepsilon} \sigma^{\cdot} \cdot \sigma_{i}^{n}}\left[\left\|\sum_{\pi}\left[x\left(s_{i}\right)-x_{n}\left(s_{i}\right)\right] \alpha\left(\sigma \cdot \sigma_{i}{ }^{n}\right)\right\|_{U}\right. \\
& \left.+\left\|\sum_{\pi} x_{n}\left(s_{i}\right) \alpha\left(\sigma \cdot \sigma_{i}^{n}\right)-J\left(x_{n}, \sigma\right)\right\|_{U}+\left\|J\left(x_{n}, \sigma\right)-J(\sigma)\right\|_{U}\right] \\
& <\underset{\boldsymbol{s}_{i \varepsilon} \sigma^{\circ \cdot \sigma_{i}}}{\operatorname{lin}}\left[\sum_{\pi}\left\|x\left(s_{i}\right)-x_{n}\left(s_{i}\right)\right\|_{U} \alpha\left(\sigma \cdot \sigma_{i}{ }^{n}\right)+1 / 2^{n}+1 / 2^{n}\right] \\
& \leqq \frac{\alpha(\sigma)}{2^{n} \alpha(S)}+\frac{1}{2^{n-1}} \leqq \frac{1}{2^{n-2}}
\end{aligned}
$$


Statement (3) postulates for a given $U$, a $\delta_{U}>0$ such that if $\alpha(\sigma)<\delta_{U}$ then $\left\|\int_{\sigma} x_{n} d \alpha\right\|_{U}<1 / 2^{3}$ for all $n$. We now choose $n_{0}>5$ such that $\alpha\left(\tau_{n_{0}}\right)<\delta_{U}$. Let $\bar{\sigma}_{k}^{n}=\sigma_{k}{ }^{n} \cdot\left(\tau_{n-1}-\tau_{n}\right)$ for $n>n_{0}$ and let $\bar{\Delta}$ be the subdivision $\left(\bar{\sigma}_{k}^{n}\right)$ of $\tau_{n_{0}}$. Then for any finite set $n_{i}, k_{i},\left\|\sum_{i=1}^{p} x\left(\bar{\sigma}_{k_{i}}{ }^{n i}\right) \alpha\left(\bar{\sigma}_{k_{i}}{ }^{n i}\right)\right\|_{U}<1 / 2$. This can be demonstrated as follows: By the inequality (1), if $\pi \geqq \pi_{n}$, then

$$
\left\|\sum_{x}\left[x\left(\bar{\sigma}_{k}^{n}\right) \alpha\left(\bar{\sigma}_{k}^{n}\right)-x\left(\bar{\sigma}_{k}^{n}\right) \alpha\left(\bar{\sigma}_{k}^{n}\right)\right]\right\|_{U}<\frac{1}{2^{n-3}}
$$

and a fortiori

$$
\left\|\sum_{n_{i}=n}\left[x\left(\bar{\sigma}_{k_{i}}^{n_{i}}\right) \alpha\left(\bar{\sigma}_{k_{i}}^{n_{i}}\right)-x\left(\bar{\sigma}_{k_{i}}^{n_{i}}\right) \alpha\left(\bar{\sigma}_{k_{i}}^{n_{i}}\right)\right]\right\|_{U}<\frac{1}{2^{n-3}}
$$

Therefore

$$
\left\|\sum_{i=1}^{p}\left[x\left(\bar{\sigma}_{k_{i}}^{n_{i}}\right) \alpha\left(\bar{\sigma}_{k_{i}}^{n_{i}}\right)-x\left(\bar{\sigma}_{k_{i}}^{n_{i}}\right) \alpha\left(\bar{\sigma}_{k_{i}}^{n_{i}}\right)\right]\right\|_{U}<\frac{1}{2^{n_{0}-3}} \leqq \frac{1}{2^{3}}
$$

Let $l$ be the greater of $n_{i}(i=1,2, \cdots, p)$. Clearly there exists $\pi^{\prime} \geqq \pi_{l}$ for which

$$
\left\|\left[\sum_{t \varepsilon \pi} \alpha\left(\sigma_{t}^{l} \cdot \bar{\sigma}_{k_{i}}^{n_{i}}\right)-\alpha\left(\bar{\sigma}_{k_{i}}^{n_{i}}\right)\right] x\left(\bar{\sigma}_{k_{i}}^{n_{i}}\right)\right\|_{U}<\frac{1}{2^{3} p}
$$

whenever $\pi \geqq \pi^{\prime}$. Hence if $\pi \geqq \pi^{\prime}$,

$$
\begin{aligned}
\sum_{i=1}^{p}\left\{\left[\sum_{t \varepsilon \pi} x\left(\sigma_{t}^{l} \cdot \bar{\sigma}_{k_{i}}^{n_{i}}\right) \alpha\left(\sigma_{t}^{l} \cdot \bar{\sigma}_{k_{i}}^{n_{i}}\right)\right]-x\left(\bar{\sigma}_{k_{i}}^{n_{i}}\right) \alpha\left(\bar{\sigma}_{k_{i}}^{n_{i}}\right)\right\} \\
\subset \sum_{i=1}^{p}\left[\sum_{t \varepsilon \pi} x\left(\sigma_{t}^{l} \cdot \bar{\sigma}_{k_{i}}^{n_{i}}\right) \alpha\left(\sigma_{t}^{l} \cdot \bar{\sigma}_{k_{i}}^{n_{i}}\right)\right]-\sum_{i=1}^{p}\left[\sum_{t \varepsilon \pi} x\left(\bar{\sigma}_{k_{i}}^{n_{i}}\right) \alpha\left(\sigma_{t}^{l} \cdot \bar{\sigma}_{k_{i}}^{n_{i}}\right)\right] \\
\quad+\sum_{i=1}^{p} x\left(\bar{\sigma}_{k_{i}}^{n_{i}}\right)\left[\sum_{t \varepsilon \pi} \alpha\left(\sigma_{t}^{l} \cdot \bar{\sigma}_{k_{i}}^{n_{i}}\right)-\alpha\left(\bar{\sigma}_{k_{i}}^{n_{i}}\right)\right] \\
=\sum_{i=1}^{p}\left\{\sum_{t \varepsilon \pi}\left[x\left(\sigma_{t}^{l} \cdot \bar{\sigma}_{k_{i}}^{n_{i}}\right)-x\left(\bar{\sigma}_{k_{i}}^{n_{i}}\right)\right] \alpha\left(\sigma_{t}^{l} \cdot \bar{\sigma}_{k_{i}}^{n_{i}}\right)\right\} \\
\quad+\sum_{i=1}^{p} x\left(\bar{\sigma}_{k_{i}}^{n_{i}}\right)\left[\sum_{t \varepsilon \pi} \alpha\left(\sigma_{t}^{l} \cdot \bar{\sigma}_{k_{i}}^{n_{i}}\right)-\alpha\left(\bar{\sigma}_{k_{i}}^{n_{i}}\right)\right] \\
\subset \sum_{i=1}^{p} \operatorname{cvx}\left[\left\{x\left(\bar{\sigma}_{k_{i}}^{n_{i}}\right)-x\left(\bar{\sigma}_{k_{i}}^{n_{i}}\right)\right\} \alpha\left(\bar{\sigma}_{k_{i}}^{n_{i}}\right)\right]+\frac{1}{2^{3}} U \\
\subset \\
\quad \frac{1}{2^{3}} U+\frac{1}{2^{3}} U \subset \frac{1}{2^{2}} U .
\end{aligned}
$$


The next to last inclusion follows because the inequality (2) holds for all subsets of $(1,2, \cdots, p)$. Finally since the subdivision $\left\{\left(\sigma_{j}{ }^{l} \cdot \bar{\sigma}_{k_{i}}{ }^{n i}\right), i=1, \cdots, p\right\}$ of $\sigma_{0}=\sum_{i=1}^{p} \bar{\sigma}_{k_{i}}{ }^{n i}$ is a repartition of the subdivision $\left(\sigma_{0} \cdot \sigma_{j}^{l}\right)$, the inequality (1) and Theorem 2.3 imply the existence of $\pi^{\prime \prime} \geqq \pi^{\prime}$ such that

$$
\| J\left(\sigma_{0}\right)-\sum_{i=1}^{p}\left\{\sum_{t \varepsilon \pi^{\prime \prime}} x\left(\sigma_{t}^{l} \cdot \bar{\sigma}_{k_{i}}^{n_{i}}\right) \alpha\left(\sigma_{t}^{l} \cdot \bar{\sigma}_{k_{i}}^{n_{i}}\right) \|_{U}<\frac{4}{2^{l-2}} .\right.
$$

As $\left\|J\left(\sigma_{0}\right)\right\|_{U}<1 / 2^{3}$, we have, after combining (3) and (4),

$$
\left\|\sum_{i=1}^{p} x\left(\bar{\sigma}_{k_{i}}^{n_{i}}\right) \alpha\left(\bar{\sigma}_{k_{i}}^{n_{i}}\right)\right\|_{U}<\frac{1}{2^{2}}+\frac{1}{2^{l-4}}+\frac{1}{2^{3}} \leqq \frac{1}{2} .
$$

We define a subdivision $\Delta_{0}=\left(\sigma_{i}\right)$ of $S$ to be the sets of $\left\{\sigma_{i}{ }^{n_{0}}\left(S-\tau_{n_{0}}\right)\right\}$ and of $\bar{\Delta}$. Let $\pi_{0}$ be the $\pi_{n_{0}}$ terms of $\left\{\sigma_{i}^{n_{0}} \cdot\left(S-\tau_{n_{0}}\right)\right\}$. Then if $\pi \geqq \pi_{0}$, we have

$$
\begin{aligned}
\left\|\sum_{\pi} x\left(\sigma_{i}\right) \alpha\left(\sigma_{i}\right)-J(S)\right\|_{U} \leqq & \sum_{\text {sets of } \Delta_{n}} x\left(\sigma_{i}\right) \alpha\left(\sigma_{i}\right)-J\left(S-\tau_{n_{0}}\right)\left\|_{U}+\right\| J\left(\tau_{n_{0}}\right) \|_{U} \\
& +\left\|\sum_{\text {sets of } \bar{\Delta}} x\left(\sigma_{i}\right) \alpha\left(\sigma_{i}\right)\right\|_{U} \\
& \leqq \frac{1}{2^{n_{0}}}+\frac{1}{2^{3}}+\frac{1}{2}<1 .
\end{aligned}
$$

$x(s)$ is therefore integrable to $J(S)$. Clearly what has been shown for $S$ could similarly be shown for any $\sigma \varepsilon \delta$.

We state the following theorem without proof:

Theorem 6.4. If $x_{n}(s)$ are $U$-integrable $(n=0,1, \cdots)$ and if $x_{n}(s) \rightarrow x_{0}(s)$ approximately, then the following are equivalent:

(1) $\lim _{n} \int_{\sigma} x_{n} d \alpha=\int_{\sigma} x_{0} d \alpha$ exists uniformly in $\sigma$.

(2) $\int_{\sigma} x_{n} d \alpha$ are uniformly absolutely continuous.

In the next theorem it is convenient to consider a pseudo-norm for the U-integrable functions defined as $\|J(x)\|_{U}=$ l.u.b. $\varepsilon \varepsilon S\|J(x, \sigma)\|_{U}$.

THEOREM 6.5. If $X$ is sequentially complete, $x(s)$ U-integrable, and $\phi(s)$ real-valued, measurable, and bounded by $M$, then $y(s)=\phi(s) x(s)$ is U-integrable and $\|J(y)\|_{U} \leqq 2 M\|J(x)\|_{U}$.

Let $\phi_{n}(s)$ be a step function with the property that $\left|\phi_{n}(s)-\phi(s)\right|<1 / n$. As $x(s)$ is $U$-integrable, there exists for a given $U \varepsilon V$ a $\Delta_{U}=\left(\sigma_{i}\right)$ such that $J_{\Delta_{U}}(x, S)$ is unconditionally summable with respect to $U$. Hence for every $k$, $\|x(s)\|_{U}$ will be bounded on $\tau_{k}=\sum_{i=1}^{k} \sigma_{i}$ so that $\left\|\left(\phi_{n}(s)-\phi(s)\right) x(s)\right\|_{U}<e$ for sufficiently large $n$ on $\tau_{k}$. Therefore $\phi_{n}(s) x(s) \rightarrow y(s)$ approximately. Suppose 
$\phi_{n}(s)=c_{i}{ }^{n}$ on $\sigma_{i}{ }^{n}$. Then by a lemma due to Dunford [2, p. 313]

$$
\begin{aligned}
\left\|\int_{\sigma} \phi_{n} x_{n} d \alpha\right\|_{U} & =\left\|\sum_{1}^{N_{n}} c_{i}^{n} \int_{\sigma \cdot \sigma_{i}{ }^{n}} x d \alpha\right\|_{U} \\
& \leqq 2 M \cdot \underset{\pi}{2 M . \text { u.b. }}\left[\left\|\sum_{\pi} \int_{\sigma \cdot \sigma_{i}{ }^{n}} x d \alpha\right\|_{U} \mid \pi \leqq\left(1,2, \cdots, N_{n}\right)\right] \\
& \leqq 2 M \cdot \underset{\sigma * \text { l.b. }}{ }\left[\left\|\int_{\sigma \cdot \sigma^{*}} x d \alpha\right\|_{U} \mid \sigma^{*} \varepsilon \delta\right]
\end{aligned}
$$

The absolute continuity of $\int_{\sigma} x d \alpha$ (Theorem 3.1) thus implies the equi-absolute continuity of the integrals $\int_{\sigma} \phi_{n} x d \alpha$. By Theorem 6.2, $y(s)$ is $U$-integrable and $\int_{0} y d \alpha=\lim _{n} \int_{\sigma} \phi_{n} x d \alpha$. The remaining part of the theorem results immediately from (1).

7. Instances of the $U$-integral. A further insight into the nature of the $U$-integral can be gained by displaying some familiar integrals as representations of the $U$-integral. We will first consider a very general theorem of this nature. To this end it will be recalled that in Theorem 1.1 we have shown that to every $U \varepsilon \cup$ there corresponds a set of linear continuous operations and a range $T_{U}$ such that $U \equiv\left[x|x \varepsilon X,| \bar{x}_{t}(x) \mid<1\right.$ for every $\left.t \varepsilon T_{U}\right]$. Further, since the relation $\geqq$ on the set of subdivisions $\Delta$ is transitive and compositive, the general limit of E. H. Moore and H. L. Smith [10, p. 103] is applicable to this range.

THEOREM 7.1. $x(s)$ is U-integrable if and only if for every $U \varepsilon V$, $\lim _{\Delta} \sum_{i=1}^{\infty} \bar{x}_{t}\left[x\left(\sigma \cdot \sigma_{i}\right)\right] \alpha\left(\sigma \cdot \sigma_{i}\right)=\bar{x}_{t}[J(x, \sigma)]$ uniformly in $t \varepsilon T_{U}$ and the $\sum_{i=1}^{\infty} \bar{x}_{t}\left[x\left(\sigma \cdot \sigma_{i}\right)\right] \alpha\left(\sigma \cdot \sigma_{i}\right)$ are absolutely convergent uniformly in $t \varepsilon T_{U}$.

The statement that for every $U$ and $\sigma$ there exist $\Delta_{U \sigma}$ and $\pi_{U_{\sigma}}$ such that if $\pi \geqq \pi_{U \sigma}$ then $\left\|\sum_{\pi} x\left(\sigma \cdot \sigma_{i}\right) \alpha\left(\sigma \cdot \sigma_{i}\right)-J(x, \sigma)\right\|_{U}<1$ is equivalent by Theorems 1.1 and 2.3 to the statement that for every $U$ and $\sigma$ there exists $\Delta_{U \sigma}$ such that if $\Delta \geqq \Delta_{U \sigma}$ there is a $\pi_{\Delta}$ such that if $\pi \geqq \pi_{\Delta}$ then

$$
\left|\sum_{x} \bar{x}_{t}\left[x\left(\sigma \cdot \sigma_{i}\right)\right] \alpha\left(\sigma \cdot \sigma_{i}\right)-\bar{x}_{t}[J(x, \sigma)]\right|<1
$$

for all $t \varepsilon T_{U}$.

Corollary 7.2. If $X$ is a Banach space with the weak neighborhood topology defined by all finite sets of elements in the adjoint space (that is, if $\left.U \equiv\left[x|x \varepsilon X,| \bar{x}_{t}(x) \mid<1, t=1, \cdots, n\right]\right)$, then the $U$-integral is the integral defined by Pettis [4, p. 280].

Each $\bar{x}$, element of the adjoint space, will clearly be continuous in the 
weak topology and hence by Corollary $5.2 \bar{x}[J(x, \sigma)]=\int_{\sigma} \bar{x}[x(s)] d \alpha$ for every $\sigma \varepsilon S$. Conversely, if the latter is true, then by Theorem 7.1, the $U$-integral exists and is equal to the Pettis integral.

Dunford [2, p. 338] has considered a more general type of integral defined as follows: Let $X$ be a closed linear manifold contained in $\bar{Y}$, the adjoint space to a Banach space $Y$. Then a function $y(s)$ on $S$ to $Y$ which has the property that $x[y(s)]$ is summable for every $x \varepsilon X$ defines uniquely an element in $\bar{X}$ according to the equation

$$
\bar{x}_{\sigma}[x]=\int_{\sigma} x[y(s)] d \alpha .
$$

$\bar{x}_{\sigma}$ is the Dunford integral of $y(s)$ over $\sigma$. However each $y \varepsilon Y$ corresponds to a unique element $\bar{x} \varepsilon \bar{X}$ through the correspondence $\bar{x}(x)=x(y)$ for all $x \varepsilon X$. The Dunford integral is unchanged when we replace the integrand $y(s)$ on $S$ to $Y$ by its correspondent $\bar{x}(s)$ on $S$ to $\bar{X}$ (that is, $\int_{\sigma} x[y(s)] d \alpha=\int_{\sigma} \bar{x}(s)[x] d \alpha$ for all $x \varepsilon X$ ). If we make this replacement, we can prove by an argument similar to that used in Corollary 7.2 the following:

Corollary 7.3. If $\bar{X}$ is adjoint to a Banach space $X$ with the weak topology on $\bar{X}$ defined by all finite sets of elements of $X$, then the $U$-integral of a function to $\bar{X}$ is the integral defined by Dunford.

Theorem 7.4. If $X$ is a Banach space and $U$ its norm topology, then the $U$-integral is the integral defined by Birkhoff.

If $x(s)$ is Birkhoff integrable, then given $e>0$ and $\sigma \varepsilon S$ there exists $\Delta_{e}$ and $J(x, \sigma)$ such that $J_{\Delta_{e}}(x, \sigma)$ is unconditionally convergent* to elements in an $e$-sphere about $J(x, \sigma)$. It is a fortiori unconditionally summable to $J(x, \sigma)$ with respect to an $e$-sphere and hence $U$-integrable to $J(x, \sigma)$. Conversely, suppose $x(s)$ is $U$-integrable. Then given $e / 2^{n}$, there exists $\Delta_{n}=\left(\sigma_{i}{ }^{n}\right)$, where $\Delta_{n+1} \geqq \Delta_{n}$, and $\pi_{n}$ such that if $\pi \geqq \pi_{n}$ then $\left\|\sum_{\pi} x\left(\sigma_{i}{ }^{n}\right) \alpha\left(\sigma_{i}{ }^{n}\right)-J(x, S)\right\|<e / 2^{n}$ $(n=0,1, \cdots)$. If $N_{n}$ is the greater of the integers in $\pi_{n}$, then $m, i_{k} \geqq N_{n}$ implies that $\left\|\sum_{1}^{m} x\left(\sigma_{i}{ }^{n}\right) \alpha\left(\sigma_{i}{ }^{n}\right)-J(x, S)\right\|<e / 2^{n}$ and $\left\|\sum_{k=1}^{p} x\left(\sigma_{i_{k}}{ }^{n}\right) \alpha\left(\sigma_{i_{k}}{ }^{n}\right)\right\|<2 e / 2^{n}$ $(n=0,1, \cdots)$. Let $P_{n}$ be the greater of $N_{n}$ and $M_{n}$ where $\sum_{i=M_{n}}^{\infty} \alpha\left(\sigma_{i}{ }^{n}\right)<1 / 2^{n}$. We define a sequence of sets $\sigma_{1}, \sigma_{2}, \cdots, \sigma_{n}, \cdots$ as follows: $\sigma_{1}=\sigma_{1}{ }^{0}$. Suppose $\sigma_{k}$ is of $\Delta_{n}$. Then $\sigma_{k+1}$ is the set of lowest subscript of $\Delta_{n}$ which is disjoint from any of the previously chosen sets unless all such sets have subscripts greater than $P_{n}$. In the latter case, $R_{n}=k$ and $\sigma_{k+1}$ is the set of lowest subscript of $\Delta_{n+1}$ which is disjoint from any of the previously chosen sets. $\sum_{i=1}^{R_{n}} \alpha\left(\sigma_{i}\right) \geqq \alpha(S)-1 / 2^{n}$.

* If $X$ is sequentially complete, then $\sum B_{i}$ where $B_{i} \subset X$ is said to be unconditionally convergent if given $U \varepsilon \mathcal{V}$ there exists $N_{U}$ such that if $i_{k} \geqq N_{U}$ then $\left\|\sum_{k_{-1}}^{p} B_{i_{k}}\right\|_{U}<1$. 
Hence $\Delta=\left(\sigma_{i}\right)$ is a subdivision of $S$. Now since $\Delta \geqq \Delta_{0}$, there exists by Theorem 2.3 an $N_{\Delta}$ such that $\left\|\sum_{1}^{n} x\left(\sigma_{i}\right) \alpha\left(\sigma_{i}\right)-J(x, S)\right\|<4 e$ if only $n \geqq N_{\Delta}$. Finally by our choice of $\left\{\Delta_{n}\right\}$, if $i_{k}>R_{n}$ then $\sigma_{i_{k}}$ is a subset of some $\sigma_{i}{ }^{n}\left(i \geqq N_{n}\right)$. Hence there exists $\pi$ such that $\pi \cdot\left(1, \cdots, N_{n}\right)=0$ and

$$
\begin{aligned}
\left\|\sum_{k=1}^{p} x\left(\sigma_{i_{k}}\right) \alpha\left(\sigma_{i_{k}}\right)\right\| & \leqq\left\|\sum_{x} \operatorname{cvx}\left[x\left(\sigma_{i}^{n}\right) \alpha\left(\sigma_{i}^{n}\right)+\theta\right]\right\| \\
& \leqq \text { l.u.b. }\left[\left\|\sum_{\pi^{\prime}} x\left(\sigma_{i}^{n}\right) \alpha\left(\sigma_{i}^{n}\right)\right\| \mid \pi^{\prime} \leqq \pi\right] \leqq \frac{e}{2^{n-1}}
\end{aligned}
$$

$J_{\Delta}(x, S)$ is therefore unconditionally convergent to elements in an $e$-sphere about $J(x, S)$ and consequently $x(s)$ is Birkhoff integrable.

In $\$ 10$ we demonstrate that the Birkhoff integral is distinct from the Pettis integral which in turn is distinct from the Dunford integral. Hence if we limit ourselves to Banach spaces on which we impose all possible convex neighborhood topologies which lie, say, in strength* between the norm topology and the usual weak topology, the corresponding ranges of integrable functions lie between the Birkhoff range and the Pettis range.

8. Differentiation. In the study of differentiation we wish to limit our attention to the subclass of $U$-integrable functions on $S=(0,1)$ to $X$ defined as follows: $x(s)$ will be said to be $V^{\prime}$-integrable if

(1) $x(s)$ is $U$-integrable.

(2) There exists a sequence of step functions $\dagger\left\{x_{n}(s)\right\}$ such that $\left\|x_{n}(s)-x(s)\right\|_{U} \rightarrow 0$ a.e. for each $U \varepsilon v$.

(3) $\|x(s)\|_{U}$ is summable for each $U \varepsilon v$.

If $X$ is a Banach space, then the $V^{\prime}$-integral is the Bochner integral [13]. On the other hand, if $S$ is a Banach space with the usual weak neighborhood topology, then the $V^{\prime}$-integral is the Pettis integral [4]. This is easily seen from Corollary 7.2 and by writing $x_{n}(s)=\int_{I_{i}}{ }^{n} x(s) d s /\left|I_{i}{ }^{n}\right|$ if $s \varepsilon I_{i}{ }^{n}$ where $I_{i}{ }^{n}=((i-1) / n, i / n)$. Thus a peculiarity of this weak neighborhood topology is that the classes of $U$ - and $V^{\prime}$-integrable functions on $(0,1)$ to $X$ are the same (see Corollary 7.2).

We define a function $x(I)$ on intervals of $(0,1)$ to $X$ to be of bounded variation $\ddagger$ if for every $U \varepsilon \cup$ there exists an $M_{U}$ such that for every finite set of disjoint intervals $I_{1}, \cdots, I_{n}, \sum_{i=1}^{n}\left\|x\left(I_{i}\right)\right\|_{U} \leqq \dot{M_{U}}$. The function $x(I)$ will

\footnotetext{
* $\mathcal{U}$ is said to be weaker than $U$ if whenever $x_{n} \rightarrow x$ in $U$ then $x_{n} \rightarrow x$ in $U$.

$\dagger$ A function on $(0,1)$ to $X$ will be called a step function if and only if it is single-valued on each of a finite number of disjoint intervals whose sum is $(0,1)$.

$\ddagger$ This is a generalization of the two notions of strong and weak bounded variation which have previously been introduced (see [3]).
} 
be said to be pseudo-differentiable* if there exists a $y(s)$ on $(0,1)$ to $X$ such that for every $U \varepsilon \mho$, a.e.

$$
\lim _{|I| \rightarrow 0}\left\|\frac{x(I)}{|I|}-y(s)\right\|_{U}=0
$$

where $I$ is an arbitrary interval containing $s$. The function $y(s)$ is the pseudoderivative of $x(I)$.

Theorem 8.1. A necessary and sufficient condition that a single-valued, additive, absolutely continuous function $x(\sigma)$ on measurable sets in $(0,1)$ to $X$ be pseudo-differentiable and of bounded variation is that the function $x(\sigma)$ be a $U^{\prime}$-integral. $x(\sigma)$ is then the $U^{\prime}$-integral of its pseudo-derivative.

Proof of the necessity. Let $x_{n}(s)=x\left(I_{i}{ }^{n}\right) /\left|I_{i}{ }^{n}\right|$ on $I_{i}{ }^{n}=\left(i / 2^{n},(i+1) / 2^{n}\right)$ $\left(i=0,1, \cdots, 2^{n}-1\right)$. Then by the definition of the pseudo-derivative there exists a $y(s)$ for which $\left\|x_{n}(s)-y(s)\right\|_{U} \rightarrow 0$ a.e. Since $\left\|x_{n}(s)\right\|_{U}$ is measurable and $\left\|x_{n}(s)\right\|_{U} \rightarrow\|y(s)\|_{U}$ a.e., it follows that $\|y(s)\|_{U}$ is measurable. As $x(\sigma)$ is of bounded variation, $\int_{\sigma}\left\|x_{n}(s)\right\|_{U} d s \leqq M_{U}$. Therefore by Fatou's lemma, $\|y(s)\|_{U}$ will be summable. It remains only to show that $y(s)$ is $U$-integrable to the value $x(\sigma)$. Now given $e>0$ and $U \varepsilon V$, there exists $\delta_{e U}>0$ such that if $|\sigma|<\delta_{e U}$ then $\int_{\sigma}\|y(s)\|_{U} d s \leqq e / 8$. Further, since $\left\|x_{n}(s)-y(s)\right\|_{U}=\lim _{m \rightarrow \infty} \| x_{n}(s)$ $-x_{m}(s) \|_{U}$ a.e. and is thus measurable, there exists $\sigma_{e U}$ such that $\left\|x_{n}(s)-y(s)\right\|_{U}$ $\rightarrow 0$ uniformly on $(0,1)-\sigma_{e U}=\tau_{e U}$ and $\left|\sigma_{\theta U}\right|<\delta_{e U}$. Let $n$ be chosen so that $\left\|x_{n}(s)-y(s)\right\|_{U}<e / 4$ for $s \varepsilon \tau_{e U}$. If $\sigma_{i}(i=1, \cdots, N)$ are the sets on which $x_{n}(s)$ is constant in $\tau_{e U}$, then

$$
\left\|\sum_{i=1}^{N} y\left(\sigma \cdot \sigma_{i}\right) \cdot\left|\sigma \cdot \sigma_{i}\right|-\sum_{i=1}^{N} x_{n}\left(\sigma \cdot \sigma_{i}\right) \cdot\left|\sigma \cdot \sigma_{i}\right|\right\|_{U}<e / 4
$$

Finally let $\sigma_{i}(i=N+1, \cdots)$ be a subdivision of $\sigma_{e U}$ for which

$$
\left\|\sum_{N+1}^{\infty}\right\| y\left(\sigma_{i}\right)\left\|_{U}\left|\sigma_{i}\right|-\int_{\sigma_{e U}}\right\| y(s)\left\|_{U} d s\right\| \leqq e / 8 .
$$

Then for $i_{k}>N$,

$$
\begin{aligned}
\left\|\sum_{k=1}^{p} y\left(\sigma \cdot \sigma_{i_{k}}\right) \cdot\left|\sigma \cdot \sigma_{i_{k}}\right|\right\|_{U} & \leqq \sum_{k=1}^{p}\left\|y\left(\sigma \cdot \sigma_{i_{k}}\right)\right\|_{U} \cdot\left|\sigma \cdot \sigma_{i_{k}}\right| \leqq \sum_{N+1}^{\infty}\left\|y\left(\sigma_{i}\right)\right\|_{U}\left|\sigma_{i}\right| \\
& \leqq e / 8+\int_{\sigma_{e U}}\|y(s)\|_{U} d s \leqq e / 4
\end{aligned}
$$

\footnotetext{
* Pettis has used this definition of pseudo-differentiability for a weak neighborhood topology on a Banach space.
} 
For $\Delta=\left(\sigma_{i}\right)$ and $\pi_{i} \geqq(1,2, \cdots, N)$ we have

$$
\left\|\sum_{\pi_{1}} y\left(\sigma \cdot \sigma_{i}\right)\left|\sigma \cdot \sigma_{i}\right|-\sum_{\pi_{2}} y\left(\sigma \cdot \sigma_{i}\right)\left|\sigma \cdot \sigma_{i}\right|\right\|_{U}<e .
$$

Now by Theorem 1.1 there exist linear continuous operations $\bar{x}_{t}$ on $X$ such that $U \equiv\left[x|x \varepsilon X,| \bar{x}_{t}(x) \mid<1\right.$ for every $\left.t \varepsilon T_{U}\right]$. Our hypothesis clearly implies $\bar{x}_{t}[x(\sigma)]=\int_{\sigma} \bar{x}_{t}[y(s)] d s{ }^{*}$ There will therefore exist a subdivision $\Delta_{t}=\left(\sigma_{i}{ }^{t}\right) \geqq \Delta$ for which

$$
\left|\sum_{i} \bar{x}_{t}\left[y\left(\sigma \cdot \sigma_{i}{ }^{t}\right)\right] \cdot\right| \sigma \cdot \sigma_{i}{ }^{t}\left|-\bar{x}_{t}[x(\sigma)]\right|<e .
$$

By (1) if $J_{t}(\sigma)$ is some element of $\sum_{x_{0}} \bar{x}_{t}\left[y\left(\sigma \cdot \sigma_{i}\right)\right]\left|\sigma \cdot \sigma_{i}\right|$ where $\pi_{0} \equiv(1,2, \cdots, N)$, then $\left|\bar{x}_{t}\left[\sum_{\pi} y\left(\sigma \cdot \sigma_{i}\right)\left|\sigma \cdot \sigma_{i}\right|\right]-J_{t}(\sigma)\right|<e$ for $\pi \geqq \pi_{0}$. Theorem 2.3 then implies

$$
\left|\sum_{i} \bar{x}_{t}\left[y\left(\sigma \cdot \sigma_{i}{ }^{t}\right)\right] \cdot\right| \sigma \cdot \sigma_{i}{ }^{t}\left|-J_{t}(\sigma)\right|<4 e
$$

and hence $\left|J_{t}(\sigma)-\bar{x}_{t}[x(\sigma)]\right|<5 e$. Therefore for $\pi \geqq \pi_{0}$,

$$
\left|\bar{x}_{t}\left[\sum_{x} y\left(\sigma \cdot \sigma_{i}\right)\left|\sigma \cdot \sigma_{i}\right|-x(\sigma)\right]\right|<6 e
$$

for every $t \varepsilon T_{U}$ or, what amounts to the same thing,

$$
\left\|\sum_{\pi} y\left(\sigma \cdot \sigma_{i}\right) \cdot\left|\sigma \cdot \sigma_{i}\right|-x(\sigma)\right\|_{U}<6 e .
$$

$y(s)$ is thus integrable to the value $x(\sigma)$.

Proof of the sufficiency. We will first prove a lemma.

LEMмA 8.2. If $x(s)$ is U-integrable and $\|x(s)\|_{U}$ is integrable for a given $U \varepsilon V$, then $\left\|\int_{\sigma} x d \alpha\right\|_{U} \leqq \int_{\sigma}\|x(s)\|_{U} d \alpha$.

Given $e>0$, there will be a $\Delta_{e}$ and $\pi_{e}$ such that

$$
\begin{gathered}
\left\|\sum_{\pi_{e}} x\left(\sigma \cdot \sigma_{i}\right) \alpha\left(\sigma \cdot \sigma_{i}\right)-J(x, \sigma)\right\|_{U}<e / 2, \\
\left|\sum_{\pi_{\theta}}\left\|x\left(\sigma \cdot \sigma_{i}\right)\right\| \alpha\left(\sigma \cdot \sigma_{i}\right)-\int_{\sigma}\|x(s)\|_{U} d \alpha\right|<e / 2 .
\end{gathered}
$$

* If $x(\sigma)$ defined only on intervals is additive, absolutely continuous, of bounded variation, and has a pseudo-derivative, then this statement and the rest of the necessity remains valid for integrals on intervals. If $X$ is in addition complete with respect to $D$, the integral can be extended on all measurable sets. 
Since we have $\left\|\sum_{\pi_{e}} x\left(\sigma \cdot \sigma_{i}\right) \alpha\left(\sigma \cdot \sigma_{i}\right)\right\|_{U} \leqq \sum_{\pi_{e}}\left\|x\left(\sigma \cdot \sigma_{i}\right)\right\|_{U} \alpha\left(\sigma \cdot \sigma_{i}\right)$, it follows that $\|J(x, \sigma)\| \leqq \int_{\sigma}\|x(s)\|_{U} d \alpha+e$ where the $e$ is arbitrary.

The proof of the sufficiency is now essentially an argument used by Bochner [13, pp. 269-270]. As $y(s)$ is $V^{\prime}$-integrable, there will exist a sequence $x_{n}{ }^{*}(s)$ of step functions such that $\left\|x_{n}^{*}(s)-y(s)\right\|_{U} \rightarrow 0$ a.e. Define $x_{n}(s)=x_{n}^{*}(s)$ if $\left\|x_{n}^{*}(s)\right\|_{U} \leqq\|y(x)\|_{U}+1, x_{n}(s)=0$ otherwise. Then $\left\|x_{n}(s)-y(s)\right\|_{U} \rightarrow 0$ a.e.; $\left\|x_{n}(s)-y(s)\right\|_{U}$ is bounded by the summable function $\left(2\|y(s)\|_{U}+1\right)$; and $\left\|x_{n}(s)-y(s)\right\|_{U}$ is measurable since $\left\|x_{n}(s)-y(s)\right\|_{U}=\lim _{m}\left\|x_{n}(s)-x_{m}(s)\right\|_{U}$. Hence making use of the lemma,

$$
\left\|\int_{\sigma}\left[x_{n}(s)-y(s)\right] d s\right\|_{U} \leqq \int_{\sigma}\left\|x_{n}(s)-y(s)\right\|_{U} d s .
$$

Finally $\int_{\sigma} x_{n}(s) d s$ has a pseudo-derivative because $x_{n}(s)$ assumes only a finite set of values. Therefore

$$
\begin{aligned}
\lim _{|I| \rightarrow 0}\left\|\frac{\int_{I} y(s) d s}{|I|}-y(s)\right\| & \leqq \lim _{|I| \rightarrow 0} \frac{\int_{I}\left\|y(s)-x_{n}(s)\right\|_{U} d s}{|I|} \\
& +\lim _{|I| \rightarrow 0}\left\|\frac{\iint_{I} x_{n}(s) d s}{|I|}-x_{n}(s)\right\|\left\|_{U}+\right\| x_{n}(s)-y(s) \|_{U} \\
& \leqq 2\left\|x_{n}(s)-y(s)\right\|_{U} \text { a.e. }
\end{aligned}
$$

As $\lim _{n}\left\|x_{n}(s)-y(s)\right\|_{U}=0$ a.e., $y(s)$ is the pseudo-derivative of $x(\sigma)$. Bounded variation follows from the lemma.

This theorem has previously been proved for the special cases where $X$ is a Banach space $[14$, p. 410 , footnote] and where $X$ is a Banach space with its weak neighborhood topology [4, p. 300].

We may generalize the notion of derivative in another way: namely, a function $x(I)$ on intervals of $(0,1)$ to $X$ is said to be differentiable at a point $s$ if $y(s) \varepsilon X$ exists such that for every $U$

$$
\lim _{|I| \rightarrow 0}\left\|\frac{x(I)}{|I|}-y(s)\right\|_{U}=0
$$

where $I$ is an arbitrary interval containing $s . y(s)$ is called the derivative of $x(I)$ at $s$. The vanishing of the limit is here independent of the choice of $U$. This kind of derivative does not seem to lend itself to a theorem analogous to Theorem 8.1. One should expect the integral of a well behaved integrand to have a derivative a.e. However in Example 10.9 we exhibit an additive, absolutely continuous function on measurable sets of $(0,1)$ to a Hilbert space with its weak neighborhood topology which is pseudo-differentiable and of 
bounded variation but not differentiable a.e. On the other hand its pseudoderivative is both $V^{\prime}$-integrable and the limit in the norm sense of a sequence of step functions.

Pettis [23] has treated in detail the case $x(I)$ of bounded variation in the norm topology on a Banach space. In some special cases we have been able to demonstrate a few propositions about the derivative in a weak neighborhood topology. Theorem 5.1 of Pettis' paper is similar to Theorem 8.4 below.

In the remainder of this section $X_{1}$ will designate a Banach space and $X$ the space $X_{1}$ with its weak neighborhood topology defined by all finite sets of linear limited operations on $X_{1}$.

$x(I)$ will be said to satisfy the weak Lipschitz condition (w.L.) if a.e. on $(0,1)$ the norm of $x(I) /|I|$ is not infinite (that is, $\|x(I) /|I|\|<\infty)$ for all intervals containing $s \varepsilon(0,1)$.

THEOREM 8.3. If $x(I)$ on intervals to $X$ is differentiable a.e., then it satisfies $w . L$.

As the neighborhoods of $X$ are defined by all finite sets of elements of $\bar{X}_{1}$, adjoint to $X_{1}$, we have a.e. $\lim _{|I| \rightarrow 0} \bar{x}[x(I) /|I|]$ exists and a fortiori $|\bar{x}[x(I) /|I|]|<\infty$ for arbitrary $I \supset s$ and all $\bar{x} \varepsilon \bar{X}_{1}$. The theorem is then an immediate consequence of a theorem due to Dunford on uniform boundedness [2, p. 308, Theorem 2]. In this case the abstract set is the set of all intervals containing a point $s$ at which the derivative exists.

THEOREM 8.4. If $X$ is sequentially complete with $\bar{X}$ separable or if $X_{1}$ is regular and if $x(I)$ on intervals to $X$ is of bounded variation, then a necessary and sufficient condition that $x(I)$ be differentiable a.e. is that $x(I)$ satisfy w.L.

The necessity has been proved in Theorem 8.3 . For the sufficiency argument we first suppose $\bar{X}_{1}$ to be separable and obtain a sequence $\left\{\bar{x}_{p}\right\}$ dense in $\bar{X}_{1}$. Since $\bar{x}_{p}[x(I)]$ is of bounded variation, $\lim _{|I| \rightarrow 0} \bar{x}_{p}[x(I)] /|I|$ exists on a set $\sigma_{p}$ of measure one. W.L. implies the existence of a function $L(s)$ such that $\bar{x}[x(I)] /|I|<L(s)\|\bar{x}\|$ on a set $\sigma^{\prime}$ of measure one. Therefore $\lim _{|I| \rightarrow 0} \bar{x}_{p}[x(I)] /|I|$ exists uniformly in $p$ on a set $\sigma_{0}=\sigma^{\prime} \cdot \prod \sigma_{p}$ of measure one. It follows that $\lim _{|I| \rightarrow 0} \bar{x}[x(I)] /|I|$ exists on $\sigma_{0}$ for all $\bar{x} \varepsilon \bar{X}_{1}$. As $X$ is sequentially complete, the derivative likewise exists on $\sigma_{0}$. If $X_{1}$ is regular and separable, then $\bar{X}_{1}$ is likewise separable. There remains only the case for which $X_{1}$ is a nonseparable regular Banach space. But here the above argument suffices because (1) $x(I)$ is contained in a separable closed linear subspace of $X_{1}$ and (2) every closed linear subspace of $X_{1}$ is regular [15, p. 423, Theorem 3]. (1) is a consequence of the following lemma: 
LEMMA 8.5. If $x(s)$ on $(0,1)$ to a sequentially complete space $X$ is of bounded variation, then $x(s)$ can have at most a denumerable number of points of discontinuity relative to the norm.

Suppose the contrary to be true. Then there exists a positive number $e$ and a denumerable set of points at which the norm variation of $x(s)$ is greater than $e$. We can then find a denumerable sequence of pairs of points with the property that $\left\|x\left(s_{n}{ }^{1}\right)-x\left(s_{n}{ }^{2}\right)\right\|>e$ and $s_{n}{ }^{1}<s_{n}{ }^{2}<s_{n+1}{ }^{1}$ (or $\left.s_{n}{ }^{1}>s_{n}{ }^{2}>s_{n}{ }^{1}+1\right)$. Let $y_{n}=x\left(s_{n}{ }^{1}\right)-x\left(s_{n}^{2}\right)$. Since $x(s)$ is of bounded variation, $\sum_{n=1}^{\infty}\left|\bar{x}\left[y_{n}\right]\right|<\infty$ for every $\bar{x} \varepsilon \bar{X}_{1}$. The sequential completeness of $X$ implies that every subseries of $\sum_{n} y_{n}$ is convergent in $X$. By a theorem due to Orlicz and Banach [4, pp. 281282, Theorem 2.32], $\sum y_{n}$ is unconditionally convergent in $X_{1}$ and hence $\left\|y_{n}\right\| \rightarrow 0$, which is contrary to our choice of $y_{n}$ 's.

Returning to the theorem, it is clear from the lemma that $x(s)=x\left(I_{0}{ }^{s}\right)$ has only a denumerable set of points of discontinuity relative to the norm and hence all of its values can be found as limits of a denumerable set of values (again in the sense of the norm). $x(I)$ is therefore contained in the separable linear closed subspace of $X_{1}$ determined by this denumerable set of values of $x(s)$. This completes the proof of Theorem 8.4.

COROLlaRy 8.6. If $X$ is sequentially complete with $\bar{X}_{1}$ separable or if $X_{1}$ is regular, and if $x(I)$ is additive, absolutely continuous, of bounded variation, and of w.L., then $x(I)$ is an integral. 301].

This follows from Theorem 8.4 and a theorem due to Pettis [4, pp. 300-

In the final theorem of this section we suppose $X_{1}$ to be a Banach space with a base $\left\{x_{i}\right\}[6$, p. 110] which satisfies the following postulate:

(M) If $a_{1}, a_{2}, \cdots$ is any sequence of real numbers such that l.u.b.n $\left\|\sum_{\mathrm{i}}^{n} a_{i} x_{i}\right\|<\infty$, then $\sum_{1}^{\infty} a_{i} x_{i}$ converges.

By redefining the norm we can consider instead of $X_{1}$ a space isomorphic $\left[6\right.$, p. 180] to $X_{1}$ satisfying in addition to $(M)$ the property

$$
\left\|\sum_{1}^{n} a_{i} x_{i}\right\| \leqq\left\|\sum_{1}^{n+1} a_{i} x_{i}\right\|
$$

for any constants $a_{1}, a_{2}, \cdots\left[16\right.$, pp. 415-416]. The spaces $l_{p}(p \geqq 1)$ and $L_{p}$ $(p>1)$ satisfy $(\mathrm{M})$ [16, p. 419].

THEOREM 8.7. If $x(I)$ is an additive, absolutely continuous function satisfying w.L. on intervals of $(0,1)$ to $X$ where $X_{1}$ satisfies $(\mathrm{M})$ and $(\mathrm{N})$, then $x(I)$ is pseudo-differentiable and is the integral of its pseudo-derivative on intervals.

Now $x(I)=\sum_{1}^{\infty} a_{i}(I) x_{i}$ where $a_{i}(I)=\bar{x}_{i}[x(I)]$ and hence are additive abso- 
lutely continuous functions of intervals with summable derivatives $a_{i}^{\prime}(s)$ a.e. As $x(I)$ satisfies w.L.,

$$
\left\|\sum_{i=1}^{n} \frac{a_{i}(I)}{|I|} x_{i}\right\| \leqq\left\|\frac{x(I)}{|I|}\right\| \leqq L_{s} \text { a.e. }
$$

so that $\left\|\sum_{1}^{\infty} a_{i}^{\prime}(s) x_{i}\right\| \leqq L_{\mathrm{s}}$ a.e. By property $(\mathrm{M}) y(s)=\sum_{1}^{\infty} a_{i}^{\prime}(s) x_{i}$ exists a.e. Let $x_{n}(I)=\sum_{1}^{n}\left[\int_{I} a_{i}^{\prime}(s) d s\right] x_{i}$. Then $\|x(I)\| \geqq\left\|x_{n}(I)\right\|$ implies that the $x_{n}(I)$ are uniformly absolutely continuous. Hence

$$
\begin{aligned}
\bar{x}[x(I)] & =\lim _{n} \bar{x}\left[x_{n}(I)\right]=\lim _{n} \int_{I}\left[\sum_{1}^{n} a_{i}{ }^{\prime}(s) \bar{x}\left(x_{i}\right)\right] d s \\
& =\int_{I}\left[\sum_{1}^{\infty} a_{i}{ }^{\prime}(s) \bar{x}\left(x_{i}\right)\right] d s=\int_{I} \bar{x}[y(s)] d s .
\end{aligned}
$$

This is equivalent to the desired conclusions.

9. An application to differential equations. We consider in this section an existence theorem for ordinary differential equations in $X$ similar to a real variable theorem due to Carathéodory $[17$, p. 672].* We limit ourselves to linear convex topological spaces which are sequentially complete and satisfy the first countability axiom. Garrett Birkhoff [17] has shown that such a space has a group metric. $X$ is therefore an $F$-space [6, p. 34] with a convex neighborhood topology. We will describe the topology by both a metric and the convex neighborhoods as suits our convenience.

$x(s)$ and $\phi(x, s)$ will be functions on $(0,1)$ and the product space $X \times(0,1)$ respectively to $X . D x(s)$ will designate the pseudo-derivative of $x(s)$. Since $X$ satisfies the first countability axiom, the existence of the pseudo-derivative will imply the existence of the derivative a.e. (see $\$ 8$ ). $\int_{\sigma} x(s) d s$ will be the $V^{\prime}$-integral. The system

$$
D x=\phi(x, s) \quad x(0)=x_{0}
$$

will be said to have a solution $x(s)$ if and only if

$$
x(s)=x_{0}+\int_{0}^{s} \phi[x(t), t] d t .
$$

$x(s)$ will be called measurable if there exists a sequence of step functions $\left\{x_{n}(s)\right\}$ such that $\left|x_{n}(s)-x(s)\right| \rightarrow 0$ a.e. where $|x-y|$ is the distance between $x$ and $y$. We will say that the sequence of functions $\left\{x_{n}(s)\right\}$ are equally-continuous if for every $U \varepsilon \mho$ there exists $\delta_{U}>0$ such that $\left|s_{1}-s_{2}\right|<\delta_{U}$ implies

\footnotetext{
* The basic ideas of this theorem were also in part suggested by T. H. Hildebrandt.
} 
$\left\|x\left(s_{1}\right)-x\left(s_{2}\right)\right\|_{U}<1$. Finally $\phi(x, s)$ will be said to satisfy condition (A) if it is continuous in $x$ for fixed $s$ and measurable in $s$ for fixed $x$.

TheOREM 9.1. A necessary and sufficient condition that $x(s)$ be a solution of (1) is that (a) $x(0)=x_{0}$, (b) $x\left(I_{s_{1}}^{s_{2}}\right)=x\left(s_{2}\right)-x\left(s_{1}\right)$ be an additive, absolutely continuous function of bounded variation on intervals of $(0,1)$ to $X$, and (c) $D x(s)$ exist and be equal to $\phi[x(s), s]$.

This is an immediate consequence of Theorem 8.1 and the footnote on page 133.

Lemma 9.2. If $\phi(x, s)$ satisfies (A) and $x(s)$ is measurable on $(0,1)$ to $X$, then $\phi[x(s), s]$ is measurable.

Since $x(s)$ is measurable, there exists a sequence of step functions $\left\{x_{n}(s)\right\}$ such that $\left|x_{n}(s)-x(s)\right| \rightarrow 0$ a.e. and by the continuity of $\phi(x, s)$ in $x$, $\left|\phi\left[x_{n}(s), s\right]-\phi[x(s), s]\right| \rightarrow 0$ a.e. Now $\phi\left[x_{n}(s), s\right]$ is clearly measurable. There will therefore exist a double sequence of step functions $\left\{y_{n}^{p}(s)\right\}$ such that for every $n, \lim _{p}\left|y_{n}{ }^{p}(s)-\phi\left[x_{n}(s), s\right]\right|=0$ a.e. As the real-valued functions $\left|y_{n}{ }^{p}(s)-\phi\left[x_{n}(s), s\right]\right|$ are measurable, there will exist the set $y_{n}{ }^{p_{n}}$ for which $\left|y_{n}{ }^{p_{n}}-\phi\left[x_{n}(s), s\right]\right|<1 / 2^{n}$ except on a set of measure less than $1 / 2^{n}$. Therefore $\left|y_{n}^{p_{n}}-\phi[x(s), s]\right| \rightarrow 0$, a.e.

LEMмA 9.3. If $\left\{x_{n}(s)\right\}$ on $(0,1)$ to $X$ are equally continuous and compactvalued for each $s \varepsilon(0,1)$, then there exists a subsequence $\left\{x_{n_{i}}(s)\right\}$ which converges uniformly to a continuous function on $(0,1)$ to $X$.

The proof is similar to that of the familiar Arzelas theorem and will be left to the reader.

LEMMA 9.4. If $\Gamma$ is a compact subset of $X$ and $M>0$, then the set of all elements of the type $\sum_{1}^{n} a_{i} x_{i}$ where $x_{i} \varepsilon \Gamma$ and $\sum_{1}^{n}\left|a_{i}\right| \leqq M$ form a compact set.

Let $\left\{U_{n}\right\}$ where $U_{n} \supset U_{n+1}$ be a complete neighborhood system for $\theta$ and let $e=1 / 2(M+1)$. There will exist a sequence $\left\{\eta_{i}\right\} \subset \Gamma$ and integers $N_{n}$ such that for $x \varepsilon \Gamma$ there is an $\eta_{i}\left(i \leqq N_{n}\right)$ such that $\left\|x-\eta_{i}\right\|_{U_{n}}<e$. In the $n$th step we let correspond to every $x \varepsilon \Gamma$ an $\eta_{i}\left(i \leqq N_{n}\right.$ and $\left.\left\|x-\eta_{i}\right\|_{U_{n}}<e\right)$ and then to every $\sum_{1}^{p} a_{j} x_{j}$ the element $\sum_{1}^{N_{n}} A_{n i} \eta_{i}$ where $A_{i}=\sum a_{j}$ summed over all $j$ such that $x_{j}$ corresponds to $\eta_{i}$. Let $\left\{\sum_{1}^{p_{r}} a_{j}^{r} x_{j}^{r}\right\}$ be a sequence of such elements and let $\left\{\sum_{1}^{N_{n}} A_{n i}^{r} \eta_{i}\right\}$ be their correspondents. Then there exists a subsequence $\left\{r_{k}\right\}$ for which $A_{n t}^{r} \rightarrow \bar{A}_{i}{ }^{n}\left(i=1, \cdots, N_{n}\right)$. Hence there will be an $N_{n}^{\prime}$ such that $k \geqq N_{n}^{\prime}$ implies

$$
\left\|\sum_{1}^{N_{n}} A_{n i}^{r_{k}} \eta_{i}-\sum_{1}^{N_{n}} \bar{A}_{i}^{n} \eta_{i}\right\|_{U_{n}} \leqq \sum_{1}^{N_{n}}\left|A_{n i}^{r_{k}}-\bar{A}_{i}^{n}\right| \cdot\left\|\eta_{i}\right\|_{U_{n}}<e .
$$


Clearly we can apply the diagonal process and obtain a subsequence $\left\{s_{k}\right\}$ for which $\left\|\sum_{1}^{N_{n}} A_{n i}^{s_{k}} \eta_{i}-\sum_{1}^{N_{n}} \bar{A}_{i}{ }^{n} \eta_{i}\right\|_{U_{n}}<e$ if $k \geqq N_{n}^{\prime \prime}$. Now

Therefore

$$
\left\|\sum_{1}^{p_{s_{k}}} a_{i}^{s_{k}} x_{i}^{s_{k}}-\sum_{1}^{N_{n}} A_{n i}^{s_{k}} \eta_{i}\right\|_{U_{n}}<M e .
$$

$$
\left\|\sum_{1}^{p_{s_{k}}} a_{i}^{s_{k}} x_{i}^{s_{k}}-\sum_{1}^{p_{s l}} a_{i}^{s_{l} x_{i}^{s_{l}}}\right\|_{U_{n}}<2(M+1) e
$$

if only $k, l \geqq N_{n}^{\prime \prime}$. As $X$ is sequentially complete, the sequence $\left\{\sum^{p^{s_{k}} a_{i}{ }^{{ }^{k}} x_{i}{ }^{{ }^{k}}}\right\}$ converges to an element of $X$.

We are now in a position to establish the existence theorem.

THEOREM 9.5. If $\phi(x, s)$ satisfies (A) and takes on only a compact set of values for $s \varepsilon(0,1)$ and $x \in X$, then the system (1) has a solution.

We first show that for any measurable function $x(s), \phi[x(s), s]$ can be uniformly approximated by simple functions* assuming values in $\Gamma \equiv[\phi(x, s) \mid s \varepsilon(0,1), x \varepsilon X]$. Let $\left\{U_{n}\right\}$ where $U_{n} \supset U_{n+1}$ be a complete neighborhood system for $\theta$. There will exist a sequence $\left\{\eta_{i}\right\} \subset \Gamma$ and integers $N_{n}$ such that for $x \in \Gamma$ there is an $\eta_{i}\left(i \leqq N_{n}\right)$ for which $\left\|x-\eta_{i}\right\|_{U_{k}} \leqq 1(k \leqq n)$. We now define $S_{n}{ }^{i} \equiv\left[s \mid s \varepsilon(0,1),\left\|\eta_{i}-\phi[x(s), s]\right\|_{U_{n}} \leqq 1\right], \sigma_{n}^{i}=S_{n}^{i}-\sum_{j=1}^{i-1} S_{n}{ }^{j}$ $\left(i=1, \cdots, N_{n}\right)$, and $y_{n}(s)=\eta_{i}$ on $\sigma_{n}{ }^{i}$. To show that $\left\{y_{n}(s)\right\}$ is the desired sequence we need only to demonstrate that $S_{n}{ }^{i}$ is measurable. But this follows from the fact that $\phi[x(s), s]$ is approximable by step functions.

By hypothesis, $\Gamma$ is compact. Therefore given $U \varepsilon \mathcal{V}$, there will exist $M(U)$ such that $\|\Gamma\|_{U} \leqq M(U)$. Further if $x(s)$ is measurable, then $\phi[x(s), s]$ is measurable, $\|\phi[x(s), s]\|_{U}$ is summable, and hence the $U$-integrability of $\phi[x(s), s]$ will imply its $V^{\prime}$-integrability.

Let $x_{n}(s)=x_{0}+\int_{0}^{s} \phi\left[x_{n}^{\prime}(s), s\right] d s$ where $x_{n}^{\prime}(s)=x(p / n)$ for $p / n<s \leqq(p+1) / n$ $(p=0,1, \cdots, n-1)$. The integral of $\phi\left[x_{n}^{\prime}(s), s\right]$ exists by Theorem 6.2 , since $\phi\left[x_{n}^{\prime}(s), s\right]$ is uniformly approximable by simple functions whose pseudonorms with respect to $U$ are bounded by $M(U)$. Now by Lemma 8.2

$$
\begin{aligned}
\left\|x_{n}\left(s_{1}\right)-x_{n}\left(s_{2}\right)\right\|_{U} & \leqq\left\|\int_{s_{1}}^{s_{2}} \phi\left[x_{n}^{\prime}(s), s\right] d s\right\| \int_{U} \leqq \int_{s_{1}}^{s_{2}}\left\|\phi\left[x_{n}^{\prime}(s), s\right]\right\|_{U} d s \\
& \leqq M(U) \cdot\left|s_{2}-s_{1}\right| .
\end{aligned}
$$

This clearly implies

$$
\left\|x_{n}(s)-x_{n}^{\prime}(s)\right\|_{U} \leqq M(U) / n .
$$

* A simple function on $(0,1)$ to $X$ is constant on each of a finite number of disjoint measurable sets whose sum is $(0,1)$. 
As above there exists a double sequence of simple functions $y_{p}{ }^{n}(s)$ such that $y_{p}{ }^{n}(s)=\eta_{i}$ on $\sigma_{p t}^{n} \varepsilon S\left(\eta_{i} \varepsilon \Gamma\right.$ and $\left.\sum_{i=1}^{N_{p}} \sigma_{p t}^{n}=S\right)$ and $\left\|y_{p}{ }^{n}(s)-\phi\left[x_{n}^{\prime}(s), s\right]\right\|_{U_{p}} \leqq 1$. It is clear that $\left\|y_{p}{ }^{n}(s)-\phi\left[x_{n}^{\prime}(s), s\right]\right\|_{U_{k}}$ is measurable and converges to zero as $p \rightarrow \infty$. Thus $\left\|y_{p}{ }^{n}(s)\right\|_{U} \leqq M(U)$ and $y_{p}{ }^{n}(s) \rightarrow \phi\left[x_{n}^{\prime}(s), s\right]$ approximately. By Theorem $6.2 \lim _{p} \int_{\sigma} y_{p}{ }^{n}(s) d s=\int_{\sigma} \phi\left[x_{n}^{\prime}(s), s\right] d s$. But $\int_{\sigma} y_{p}{ }^{n}(s) d s=\sum_{1}^{N_{p}} \eta_{i}\left|\sigma \cdot \sigma_{p i}^{n}\right|$ where $\sum_{t=1}^{N_{p}}\left|\sigma \cdot \sigma_{p i}^{n}\right| \leqq 1$. By Lemma 9.4 the set $\left[\int_{\sigma} y_{p}{ }^{n}(s) d s \mid \sigma \subset(0,1)\right.$; $n ; p=1,2, \cdots]$ is compact and therefore $\left[\int_{0}^{s} \phi\left[x_{n}^{\prime}(s), s\right] d s \mid s \varepsilon(0,1)\right.$; $n=1,2, \cdots]$ is compact. By (2) and Lemma 9.3 there exists a subsequence $\left\{x_{n_{k}}(s)\right\}$ which approaches the continuous function $x(s)$ uniformly on $(0,1)$. Then by (3) $x_{n_{k}^{\prime}}(s) \rightarrow x(s)$ uniformly on $(0,1)$. Clearly $\| \phi\left[x_{n_{k}}{ }^{\prime}(s), s\right]$ $-\phi[x(s), s)] \|_{U}$ is measurable. Therefore $\phi\left[x_{n_{k}}{ }^{\prime}(s), s\right] \rightarrow \phi[x(s), s]$ approximately as $k \rightarrow \infty$. Also $\left\|\phi\left[x_{n_{k}}{ }^{\prime}(s), s\right]\right\|_{U} \leqq M(U)$. Again by Theorem 6.2

$$
x(s)-x_{0}=\lim _{k} \int_{0}^{s} \phi\left[x_{n_{k}}^{\prime}(s), s\right] d s=\int_{0}^{s} \phi[x(s), s] d s .
$$

10. Examples. The following examples serve to illustrate some of the properties of the $U$-integral as well as the importance of various hypotheses. Example 10.2 demonstrates that the Birkhoff and Pettis integrals are different. It is therefore possible to define a class of integrals for a Banach space. Example 10.8 shows that the class of Pettis integrable functions does not include a very well behaved set of functions. Example 10.9 proves that the integral of a strongly measurable function may not be weakly differentiable. These three examples answer questions raised by Pettis [4, p. 303].

$B$ will be the space of real bounded functions $x_{t}$ on $T=(0,1)$ with norm $\left\|x_{t}\right\|=$ l.u.b.teT $\left|x_{t}\right|$. We will consider functions $x(s)$ on $S=(0,1)$ to $X$. Integration on $S=(0,1)$ is taken with respect to the Lebesgue measure function.

It will be necessary for us to refer to the following theorem whose proof can be found in another paper* by the author:

THEOREM 10.1. For every bounded additive set function $\beta(\tau)$ on all subsets of $(0,1)$ there exists a denumerable set $R(\beta)$ and a unique decomposition $\beta(\tau)=\beta_{1}(\tau)+\beta_{2}(\tau)$ such that $\beta_{i}(\tau)(i=1,2)$ are bounded additive set functions, $\beta_{1}(\tau)=\beta(R \cdot \tau)$, and $\beta_{2}(\tau)=0$ if $\tau$ is a denumerable subset of $(0,1)$.

10.2. Example of a function which is Pettis integrable but not Birkhoff integrable $(\$ 7)$. Let $x(s)$ on $S=(0,1)$ to $B$ be defined as $x(s) \equiv x_{t}(s)=1$ if $t-s=p / 2^{n}$ for $\pm p, n$ positive integers and $x_{t}(s)=0$ elsewhere. Thus on the unit square with coordinates $s$ and $t, x_{t}(s)=1$ only on a denumerable set of points on any line parallel to either coordinate axis.

\footnotetext{
* This paper will appear in the Bulletin of the American Mathematical Society.
} 
Hildebrandt [19] has shown that for every $\bar{x}$ of the space adjoint to $B$ there exists an additive bounded set function $\beta(\tau)$ on all subsets of $T=(0,1)$ such that for all $x \varepsilon B, \bar{x}(x)=\int_{T} x_{t} d \beta$. By Theorem 10.1, $\beta(\tau)=\beta_{1}(\tau)+\beta_{2}(\tau)$, where $\beta_{1}(\tau)=\beta(R \cdot \tau), R$ is a denumerable subset of $T$, and $\beta_{2}(\tau)=0$ for all denumerable subsets of $T$. Hence for each $s$

$$
\bar{x}[x(s)]=\int_{T} x_{t}(s) d \beta=\int_{T} x_{t}(s) d \beta_{1}+\int_{T} x_{t}(s) d \beta_{2}=\int_{T} x_{t}(s) d \beta_{1} .
$$

It follows that $\bar{x}[x(s)] \neq 0$ only if $x_{t}(s) \neq 0$ for $t \varepsilon R$. Let $\delta_{t} \equiv[s \mid s \varepsilon(0,1)$, $x_{t}(s) \neq 0$, fixed $\left.t\right]$ and $\delta_{0}=\sum_{t \varepsilon R} \delta_{t}$. Then $\delta_{0}$ is the subset of $S$ on which $x_{t}(s) \neq 0$ for some $t \varepsilon R$. Since both $\delta_{t}$ and $R$ are denumerable sets, $\delta_{0}$ is a denumerable subset of $S$. Therefore $\bar{x}[x(s)]=0$ a.e. and $x(s)$ is Pettis integrable to the value $x_{\sigma}=\theta \varepsilon B$ for all measurable sets $\sigma$.

On the other hand $x(s)$ is not Birkhoff integrable. Suppose the contrary were true. Then [1, Theorem 13], given $e>0$, there exist disjoint sets $\sigma_{i}$ such that $\sum \sigma_{i}=S$, the series $\sum x\left(\sigma_{i}\right) \alpha\left(\sigma_{i}\right)$ is unconditionally convergent, and $s_{i}, s_{i}^{\prime} \varepsilon \sigma_{i}$ implies $\left\|\sum_{i=1}^{\infty} x\left(s_{i}\right)\left|\sigma_{i}\right|-\sum_{i=1}^{\infty} x\left(s_{i}^{\prime}\right)\left|\sigma_{i}\right|\right\|<e$. We first suppose that for each $t$ there exists a $\sigma_{i}$ such that for all $s \varepsilon \sigma_{i}, x_{t}(s)=0$. We can then divide $T$ into sets $\theta_{i}$ where $T=\sum \theta_{i}$, and for all $t \varepsilon \theta_{i}$ and $s \varepsilon \sigma_{i}, x_{t}(s)=0$. Since $x_{t}(s)=1$ on a diagonal, $S-\sigma_{i}$ contains the set $\theta_{i}^{\prime}$ whose coordinate values are those of $\theta_{i}$. In addition $S-\sigma_{i}$ contains $\theta_{i}^{\prime \prime}$ consisting of all points of the type $s \pm p / 2^{n}$ and $s / 2^{n}$ where $s \varepsilon \theta_{i}^{\prime}$ for all integral $p$ and $n$. Now $\theta_{i}^{\prime \prime}$ is a homogeneous point set in the sense of Knopp [20, pp.411-414] so that its upper measure $\left|\overline{\theta_{i}^{\prime \prime}}\right|=1$ or 0 . As there exists an $i_{0}$ such that $\left|\overline{\theta_{i_{0}}^{\prime}}\right|>0$, it follows that $\left|S-\sigma_{i_{0}}\right| \geqq\left|\overline{\theta_{i_{0}}^{\prime \prime}}\right|=1$ which is contrary to $\left|\sigma_{i_{0}}\right|>0$. Our supposition being false, we can select a $t_{0}$ with the property that for every $i, \sigma_{i}$ contains an $s_{i}$ such that $x_{t_{0}}\left(s_{i}\right)=1$. As $\left|\sigma_{i}\right|>0$, there is also an $s_{i}^{\prime} \varepsilon \sigma_{i}$ such that $x_{t_{0}}\left(s_{i}^{\prime}\right)=0$. But this implies that ||$\sum_{i} x\left(s_{i}\right)\left|\sigma_{i}\right|-\sum_{i} x\left(s_{i}^{\prime}\right)\left|\sigma_{i}\right| \| \geqq 1$. The assumption that $x(s)$ is Birkhoff integrable has thus led to a contradiction.

10.3. Example of a $U$-integrable function for which there exists $\Delta_{0}$ such that for all $\Delta \geqq \Delta_{0}, J_{\Delta}(x, S)$ is not unconditionally convergent $(\$ 2)$. One would expect a definition of unconditional convergence for $J_{\Delta}(x, S)$ at least to demand the convergence of every sum $\sum_{1}^{\infty} x\left(s_{i}\right)\left|\sigma_{i}\right|\left(s_{i} \varepsilon \sigma_{i}\right)$. Even such a definition would eliminate the function of the present example from the class of integrable functions if (1) we replace unconditional summability in Definition 2.1 by unconditional convergence, and (2) we insist that $J_{\Delta}(x, S)$ is unconditionally convergent if this is true for $J_{\Delta_{0}}(x, S)$ where $\Delta \geqq \Delta_{0}$.

Let $X$ be the space $B$ with a weak neighborhood topology defined by all finite sets of linear limited operations on $B$. Let $x(s) \equiv x_{t}(s)=1 / s$ for $s-t=p / 2^{n}$ where $\pm p, n$ are positive integers and $x_{t}(s)=0$ elsewhere. $x(s)$ can be shown 
to be $U$-integrable (that is, Pettis integrable) in a manner identical with that used in Example 10.2. Suppose $\Delta_{0}=\left\{\sigma_{i}{ }^{0}=\left(1 / 2^{i}, 1 / 2^{i-1}\right)\right\}$ and $\Delta=\left(\sigma_{i}\right) \geqq \Delta_{0}$. As in Example 10.2 there exists $t_{0}$ and $s_{i} \varepsilon \sigma_{i}$ such that $x_{t_{0}}\left(s_{i}\right)=1 / s_{i}$. We will now show that some sum of $J_{\Delta}(x, S)$ does not converge with respect to the neighborhoods $U \equiv\left[x|x \varepsilon X,| \bar{x}_{t_{0}}(x) \mid<\delta\right]$ where $\bar{x}_{t_{0}}(x)=x_{t_{0}}$. This is an immediate consequence of

$$
\sum_{i} x_{t_{0}}\left(s_{i}\right)\left|\sigma_{i}\right| \geqq \sum_{i} \frac{1}{1 / 2^{i-1}}\left|\sigma_{i}{ }^{0}\right|=\sum_{i} \frac{2^{i-1}}{2^{i}}=\infty
$$

10.4. Example of a function $x(s)$ on $S=(0,1)$ to $B$ which is $U$-integrable having the properties (1), (2), (3) given below.

(1) $\|x(s)\|$ is not measurable. Convergence a.e. will therefore not imply uniform convergence on the complement of sets of arbitrary small measure (approximate convergence in Theorem 6.2).

(2) $\int_{\sigma} x(s) d s=\theta$ for all $\sigma$ yet $x(s) \neq 0$ a.e. $\bar{B}$ does not contain a denumerable subset which is total (Theorem 5.2).

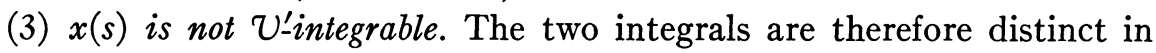
general $(\S 8)$.

Let $\sigma_{0}$ be a non-measurable set in $S=(0,1)$ and define $x(s)=\theta$ for $s \varepsilon S-\sigma_{0}$ and $x(s)$ equal to the characteristic function of the point $s$ for $s \varepsilon \sigma_{0}[4$, p. 301, Example 9.1].

10.5. Example illustrating the need for sequential completeness in Corollary 4.3 and Theorem 6.2. In the Banach space of bounded sequences let $m_{0}$ be the linear extension of the set of unit vectors $\phi_{n}$. The space $m_{0}$ is not sequentially complete. Let $x^{k}(s) \equiv x_{i}{ }^{k}(s)=\delta_{i n} \phi_{n}$ for $s \varepsilon\left(1 / 2^{n}, 1 / 2^{n-1}\right)$ and $n \leqq k\left(\delta_{i n}\right.$ is the Kronecker symbol), and vanish elsewhere $(k=1,2, \cdots, \omega)$. Despite the fact that for every $e>0$ there exists a $J_{\Delta}\left(x^{\omega}, S\right)$ which is unconditionally summable with respect to an $e$-sphere, $x^{\omega}(s)$ is not $U$-integrable in $m_{0}$ (Corollary 4.3). On the other hand $x^{k}(s) \rightarrow x^{\omega}(s)$ approximately, and the $\int_{\sigma} x^{k}(s) d s$ exist and are uniformly absolutely continuous (Theorem 6.2).

10.6. Example showing the need for sequential completeness in Theorem 6.5. Let $B_{0}$ be the linear subset of $B$ consisting of all functions on $T=(0,1)$ whose derivatives exist a.e. and take on only a finite set of values. $B_{0}$ is not sequentially complete. We define $x(s) \equiv x_{t}(s)=1$ for $t \geqq s$, and to vanish elsewhere. $x(s)$ is integrable on $\sigma$ to $\left(\left|\sigma \cdot I_{0} t\right|\right)$. However if $\phi(s)=s$, then $\int_{0}^{1} \phi(s) x(s) d s$ $=\left(t^{2} / 2\right)$ which is not in $B_{0}$.

10.7. Example showing the need of completeness with respect to $D$ in a space which is sequentially complete (Theorem 4.1). Let $l_{0}$ be the linear space of functions $x_{t}$ on $T=(0,1)$ such that $\sum_{t}\left|x_{t}\right|<\infty$ and let $\bar{l}_{0}$ be the set of all 
bounded measurable functions $\bar{x}_{t}$ on $T=(0,1)$. Then $\bar{x}(x) \equiv \sum \bar{x}_{t} \cdot x_{t}$ is a linear continuous operation on $l_{0}$. We will define the topology on $l_{0}$ by all finite sets of elements from $\bar{l}_{0}$. Any denumerable sequence of elements of $l_{0}$ differs on $T$ at only a denumerable set of points. $l_{0}$ is sequentially complete since this is equivalent to the weak completeness of $l_{1}\left[6\right.$, p. 143]. Finally set $x_{t}(s)=\delta_{s t}$ on $S=(0,1)$ to $l_{0}$. As $\int_{\sigma} \bar{x}\left(x_{t}(s)\right) d s=\int_{\sigma} \bar{x}_{t} d t$ exists, it follows that for every $U \varepsilon \mathcal{\varepsilon}$ (finite set of $\bar{x}$ 's) there will be a $J_{\Delta}(x, S)$ which is unconditionally summable with respect to $U$. On the other hand there exists no $x^{0} \varepsilon l_{0}$ such that $\sum_{t} \bar{x}_{t} \cdot \bar{x}_{t}^{0}=\int_{0}^{1} \bar{x}_{t} d t$ for all $\bar{x} \varepsilon \bar{l}_{0} . x(s)$ is therefore not $U$-integrable.

For convenience let $M$ be the class of functions $x(s)$ on $S=(0,1)$ to $B$ such that (1) $\bar{x}[x(s)]$ as measurable for all $\bar{x} \varepsilon \bar{B}$, and (2) $\int_{\sigma} \bar{x}[x(s)] d s$ are uniformly absolutely continuous with respect to elements of $\bar{B}$. If $x(s)$ is contained in a separable subspace of $B$, then it is Pettis integrable [4, p. 293, Theorem 5.3]. It is Dunford integrable in any case.

10.8. Example of a member of $M$ which is not Pettis integrable. Sierpiński [21, pp. 9-10] has shown that equivalent to the hypothesis of the continuum is the existence of a set $\theta$ in the unit square $I$ such that on every parallel to the $s$ axis $\theta$ contains at most a denumerable set of points in $I$ while on every parallel to the $t$ axis $I-\theta$ contains at most a denumerable set of points in $I$. We define $x(s)$ on $S=(0,1)$ to $B$ so that $x(s) \equiv x_{t}(s)$ is the characteristic function of $\theta$. In the notation of Example 10.2,

$$
\bar{x}[x(s)]=\int_{T} x_{t}(s) d \beta=\int_{T} x_{t}(s) d \beta_{1}+\int_{T} x_{t}(s) d \beta_{2} .
$$

Since for any $t \varepsilon(0,1), x_{t}(s)=1$ for only a denumerable set $\delta_{t}$ in $S$, it follows that $\int_{T} x_{t}(s) d \beta_{1} \neq 0$ on at most the set $\delta_{0}=\sum_{t \varepsilon R} \delta_{t}$ which is denumerable. Let $1_{t}$ be the element of $B$ which has the value one for all $t \varepsilon T$. As $\left(1_{t}-x_{t}(s)\right)$ has only a denumerable set of nonzero values for each $s \varepsilon S$, we have $\int_{T}\left(1_{t}-x_{t}(s)\right) d \beta_{2}=0$. Hence $\bar{x}[x(s)]=\int_{T} 1_{t} d \beta_{2}$ for all $s \varepsilon\left(S-\delta_{0}\right)$ so that $\bar{x}[x(s)]$ is measurable. The integrals $\int_{\sigma} \bar{x}[x(s)] d s$ are obviously uniformly absolutely continuous with respect to elements of $\bar{B}$. Thus $x(s) \varepsilon M$.

Finally, let us suppose that there exists a $y \varepsilon B$ such that $\bar{x}(y)=\int_{s} \bar{x}[x(s)] d s$ for every $\bar{x} \varepsilon B$. For every $t_{0} \varepsilon T$ there exists an $\bar{x}_{t_{0}} \varepsilon \bar{B}$ defined by $\bar{x}_{t_{0}}\left(x_{t}\right)=x_{t_{0}}$. This set is total on $B$.

$$
y_{t_{0}}=\bar{x}_{t_{0}}(y)=\int_{S} \bar{x}_{t_{0}}\left[x_{t}(s)\right] d s=\int_{S} x_{t_{0}}(s) d s=0 .
$$

Now Banach [6, p. 30] has demonstrated the existence of $\bar{x}_{0} \varepsilon \bar{B}$ for which $\bar{x}_{0}(x)=\mathrm{L} \int_{s} x_{t} d t$ whenever $x_{t}$ is Lebesgue integrable. Our supposition then leads to 


$$
0=\bar{x}_{0}(y)=\int_{S} \bar{x}_{0}\left[x_{t}(s)\right] d s=\int_{S} 1 d s=1 .
$$

Therefore $x(s)$ is not Pettis integrable.

It is to be noticed that $F(\bar{x})=\int_{s} \bar{x}[x(s)] d s$ is weakly continuous on $\bar{B}$. Since there exists no $y \varepsilon B$ such that $F(\bar{x})=\bar{x}(y)$ for all $\bar{x} \varepsilon \bar{B}$, the Banach theorem [6, p. 131, Theorem 8] to this effect for separable Banach spaces is not in general true for nonseparable Banach spaces.

10.9. Example of an additive, absolutely continuous function $x(\sigma)$ on measurable sets of $(0,1)$ to Hilbert space $H$ which in the weak neighborhood topology is pseudo-differentiable but a.e. not differentiable (\$8). Let $x_{i, j}$ be a doubly infinite set of orthonormal vectors in $H$. We define $y_{i}(s)=2^{i} x_{i, j}$ on $\left(j / 2^{i}, 2^{-2 i}+j / 2^{i}\right)$ $j=0,1, \cdots,\left(2^{i}-1\right)$, and elsewhere set $y_{i}(s)=0$. As Birkhoff [1, p. 375, Ex. ample 2] has observed, $y(s)=\sum_{n=1}^{\infty} y_{n}(s)$ exists a.e. and is Birkhoff integrable, Pettis has shown [4] that $x(\sigma)=\int_{\sigma} y(s) d s$ is additive, absolutely continuousand in the weak neighborhood topology pseudo-differentiable. We will now demonstrate that $\|x(I) /|I|\|$ is unbounded a.e. As in Theorem 8.3, this will imply that a.e. the weak derivative does not exist. Let $s={ }_{0} a_{1} a_{2} \cdots a_{n} \cdots$ be the dyadic representation of $s \varepsilon(0,1)$. Hardy and Littlewood [22, p. 189] have proved that any finite sequence of digits will recur an infinite number of times on a set of measure one. In particular, $n$ consecutive zeros will occur infinitely often on $\sigma_{n}$ of unit measure. Then $\sigma_{0}=\prod \sigma_{n}$ is of measure one. For each point of $\sigma_{0}$ all numbers of consecutive zeros occur infinitely often. Suppose $p$ is the number of consecutive zeros not exceeding $n / 2$ which immediately proceed the $(n+1)$ st digit of ${ }_{0} a_{1} a_{2} \cdots a_{n} \cdots=s$. If $I_{n}$ is the interval ${ }_{0} a_{1} a_{2} \cdots a_{n-p} \leqq s \leqq{ }_{0} a_{1} a_{2} \cdots a_{n-p}+1 / 2^{n}$, then

$$
\begin{aligned}
x\left(I_{n}\right) & =\int_{I_{n}}\left[\sum_{n-p}^{n} y_{i}(s)\right] d s+\int_{I_{n}}\left[y(s)-\sum_{n-p}^{n} y_{i}(s)\right] d s \\
& =\sum_{n-p}^{n} x_{i, j_{i}} / 2^{i}+x^{\prime}\left(I_{n}\right)
\end{aligned}
$$

where $x^{\prime}\left(I_{n}\right)$ is orthogonal to $\left(x_{i, j_{i}}\right) \quad(i=n-p, \cdots, n)$. In this case $\left\|x\left(I_{n}\right) /\left|I_{n}\right|\right\| \geqq 2^{p}$. Since for each point of $\sigma_{0}, \lim _{n} p_{n}=\infty$, our assertion is proved.

\section{REFERENCES}

1. G. Birkhoff, Integration of functions with values in a Banach space, these Transactions, vol. 38 (1935), pp. 357-378.

2. N. Dunford, Uniformity in linear spaces, these Transactions; vol. 44 (1938), pp. 305-356.

3. I. Gelfand, Sur un lemme de la théorie des espaces linéaires, Communications de l'Institut des Sciences Mathématiques et Mécaniques de l'Université de Kharkoff et la Société Mathématique de Kharkoff, vol. 13, pp. 35-40. 
4. B. J. Pettis, On integration in vector spaces, these Transactions, vol. 44 (1938), pp. 277-304.

5. A. Kolmogoroff, Zur Normierbarkeit eines allgemeinen topologischen linearen Raumes, Studia Mathematica, vol. 5 (1934), pp. 29-33.

6. S. Banach, Théorie des Opérations Linéaires, Monografje Matematyczne, Warsaw, 1932.

7. J. von Neumann, On complete topological spaces, these Transactions, vol. 37 (1935), pp. 1-20.

8. J. V. Wehausen, Transformations in linear topological spaces, Duke Mathematical Journal, vol. 4 (1938), pp. 157-169.

9. E. H. Moore, General Analysis, Memoirs of the American Philosophical Society, vol. 1, part 2, 1939.

10. E. H. Moore and H. L. Smith, A general theory of limits, American Journal of Mathematics, vol. 44 (1922), p. 103.

11. Fréchet, Sur l'intégrale d'une fonctionelle étendue à un ensemble abstrait, Bulletin de la Société Mathématique de France, vol. 43 (1915), pp. 248-265.

12. S. Saks, Addition to the note on some functionals, these Transactions, vol. 35 (1933), p. 967.

13. S. Bochner, Integration von Funktionen deren Werte die Elemente eines Vektorraumes sind, Fundamenta Mathematicae, vol. 20 (1933), pp. 262-276.

14. J. A. Clarkson, Uniformly convex spaces, these Transactions, vol. 40 (1936), pp. 396-414.

15. B. J. Pettis, $A$ note on regular Banach spaces, Bulletin of the American Mathematical Society, vol. 44 (1938), pp. 420-428.

16. N. Dunford and A. P. Morse, Remarks on the preceding paper of James A. Clarkson, these Transactions, vol. 40 (1936), pp. 415-420.

17. C. Carathéodory, Vorlesungen über reelle Funktionen, Leipzig, 1918.

18. G. Birkhoff, A note on topological groups, Compositio Mathematica, vol. 3 (1936), pp. 427430.

19. T. H. Hildebrandt, Linear operations on functions of bounded variation, Bulletin of the American Mathematical Society, vol. 44 (1938), p. 75.

20. K. Knopp, Mengentheoretische Behandlung einiger Probleme der diophantischen Approximationen und der transfiniten Wahrscheinlichkeiten, Mathematische Annalen, vol. 95 (1925), pp. 409-420.

21. W. Sierpinski, Hypothèse du Continu, Monografje Matematyczne, Warsaw, 1934.

22. G. H. Hardy and J. E. Littlewood, Some problems of diophantine approximation, Acta Mathematica, vol. 37 (1914), pp. 155-190.

23. B. J. Pettis, Differentiation in Banach spaces, Duke Mathematical Journal, vol. 5 (1939), pp. 254-269.

UNIVERSITY OF MichIGAN, ANN ARBOR, Mich. 